\title{
Chemicapacitors as a versatile platform for miniature gas and vapor sensors (Invited Paper)
}

\author{
Robert Blue and Deepak Uttamchandani* \\ Centre for Microsystems and Photonics, University of Strathclyde, Glasgow, G11XW, UK. \\ *Correspondance: d.uttamchandani@strath.ac.uk; Tel.: +44-141-548-2543
}

\begin{abstract}
Recent years have seen the rapid growth in the need for sensors throughout all areas of society including environmental sensing, health-care, public safety and manufacturing quality control. To meet this diverse need, sensors have to evolve from specialized and bespoke systems to miniaturized, low-power, lowcost (almost disposable) ubiquitous platforms. A technology that has been developed which gives a route to meet these challenges is the chemicapacitor sensor. To date the commercialization of these sensors has largely been restricted to humidity sensing, but in this review we examine the progress over recent years to expand this sensing technology to a wide range of gases and vapors. From sensors interrogated with laboratory instrumentation, chemicapacitor sensors have evolved into miniaturized units integrated with low power readout electronics that can selectively detect target molecules to ppm and sub-ppm levels within vapor mixtures.
\end{abstract}

Keywords: miniature sensors, chemicapacitors, volatile organic chemicals (VOCs), low-power, stand-alone sensors

\section{Introduction}

The relentless release of chemical waste products globally has an adverse impact on both the surrounding environment and human health. Common inorganic air pollutants include carbon monoxide, sulphur dioxide and nitrogen dioxide primarily from the burning of fossil fuels and automobile emissions [1]. Air pollutants are linked to cancer and asthma, as well as abnormal growth and development [2-3]. Volatile organic compounds (VOCs) are a group of chemical compounds in liquid or solid form that evaporate easily at room temperature and are often in the environment in the form of vapors. Atmospheric vapors are distinct from gases in that a gas is above its critical temperature and cannot be re-liquefied under ambient conditions. Sources of VOCs can be both natural and man-made. Paints, adhesives, protective coatings, and cleaning agents contain solvents and the storage and use of these can release harmful emissions to individuals using these materials and also to building occupants. The phenomenon of "sick building syndrome", which manifests itself in headaches, nausea and respiratory problems, has been linked to air pollution by VOCs [4]. Continued long term exposure to certain VOCs can lead to more serious effects on the nervous system, to cancers, and to kidney and liver damage [5]. VOCs such as benzene, acetaldehyde, methylene chloride, and formaldehyde are of particular concern as they are proven or highly suspected carcinogens [6-8]. In recent years, regulations on the allowable concentrations of various pollutants have been introduced [9]. Guidelines define the time weighted average (TWA) exposure 
limits beyond which VOCs are considered hazardous for human health. For VOCs such as ethyl acetate, toluene, p-xylene and benzene these are 400, 200, 100 and $1 \mathrm{ppm}$ respectively [10].

In the active industrial setting, exposure to VOCs are much more common since large volumes of VOCs are essential ingredients that have to be stored and pumped through piping systems during manufacturing processes. At each part of the journey there is risk of leaks, spills and serious contamination and therefore continuous monitoring at these sites is a pre-requisite to meet strict regulations and safeguard plant and personnel. Outwith the industrial area, noxious emissions into residential areas from waste-treatment and other industrial plants have to be monitored by regulation. Areas containing waste dumps, landfill, or derelict industrial sites also require monitoring. Any leaked chemicals can take years to naturally breakdown to safe concentration levels. However, the time and cost of routine sampling of polluted land with standard equipment (or sample transport to a remote laboratory for analysis) make such, even limited, practices costly and time-consuming and minimizes the ability to continuously monitor areas of interest.

In agriculture, VOCs are released by plants in response to crop diseases [11] and in response to insect infestations [12-13], the early detection of which can help reduce the economic losses incurred. Quality control procedures in the pharmaceutical and food industries also routinely sample and analyze gas concentrations from perishable goods. The ripeness of fruits and vegetables is accompanied by the release of VOCs [14] and typical gas sample analysis may employ an expensive mass spectrometer. Elevated levels of VOCs within sealed medicine and food packaging may be indicative of compromised seals. Identifying and measuring the concentration of gas molecules also has important applications in the field of medical diagnosis [15]. In addition, miniature low-cost stand-alone sensors are also highly desirable in the area of security for detecting explosive compounds [16] or warfare agents in venues of open access to give warning and time to evacuate an area.

However, VOCs are commonly detected in vapor form, and established vapor sensors are bulky, expensive, with high power consumption, which limits their widespread use, even in an industrial setting. The rising demand for sensors throughout society has led to investigation of alternative lowcost low-power sensor devices with adequate sensitivity to target molecules (to ppm concentrations or below) and which can operate unattended. Another key requirement for this type of sensor is to operate in ambient conditions with the ability to detect target molecules within a complex gas or vapor mixture, i.e. high selectivity is desired.

\subsection{Gas and Vapor Detection Technologies-A Brief Overview}

Laboratory-based gas measurements often employ optical spectroscopy for high selectivity and sensitivity [17]. However the basic cost of fixed or tunable optical sources (often a mid-infrared laser), the requirement of adequate optical pathlength, and a suitable optical detector with high responsivity and low noise means these systems are usually of higher cost, power hungry, and less amenable to miniaturization. Thus such sensors have found limited deployment in the wider society and are often developed for a specific application and location. Ion mobility spectrometry is a very sensitive technique that detects ionized gaseous molecules according to their drift time in a carrier gas under the influence of an applied electric field $[18,19]$. Portable systems have been developed but they are still bulky, require an operator, and have a battery life of a few hours [20]. Acoustic gas 
sensors measure the velocity of sound through a target gas with respect to an in-built reference gas sample to estimate the concentration of the target gas [21]. These sensors usually have large power consumption and require a predetermined reference gas or mixture of gases. Calorimetric gas sensors use resistance changes in a ceramic element due to the presence of a gas [22]. The gas is often heated or burned leading to a sensor that dissipates significant power during operation. These types of sensors often suffer from selectivity problems within a mixture of gases as well as detection sensitivity limited to parts per thousand of the target gas. The change in conductivity of metal oxide semiconductors (MOS) in the presence of a gas is also used as the basis for a range of gas sensors [23]. However, to achieve high sensitivity the MOS is heated to a temperature of several hundred degrees during the sensor operation. This adds to the cost, power-consumption and complexity of this type of sensor.

In contrast to these technologies lower power sensors can be created by making standard capacitance measurements on thin dielectric films (often off-the-shelf polymers) whose intrinsic properties change upon absorption of a wide range of VOCs and gases. Changes in the film mass have also been measured using cantilevers [24], microbalances [25], and surface acoustic waves [26]. However, the measurement of capacitance changes negates the need for optical sources or resonance excitation and thus facilitating miniaturization. The diverse range of gases and VOCs that are required to be detected and measured using a distributed network of low-cost sensors can potentially be achieved by the development of a single low-cost technology platform, namely miniaturized sensing capacitors, which can be integrated with low-power electronics.

\section{Capacitor Sensor Topologies and Methods}

There are two basic formats of capacitance sensors, namely interdigitated electrode capacitors (see Figure 1(a)) and parallel plate capacitors (see Figure 1(b)). These platforms can be used to sense a wide range of target molecules by the deposition or growth of a thin sensing film upon the IDEs or between the plates of the parallel format. The sensing film is a receptor for the molecules being detected and its electrical properties or dimensions are modified in the presence of the target molecules.

Interdigitated electrodes (IDEs) are commonly formed on an inert substrate as pairs of overlapping parallel combs with a fixed spacing between the electrodes. Traditionally, the IDEs are fabricated on solid substrates such as glass or silicon [27], but more recently flexible plastic substrates have been used [28]. These planar IDEs can be fabricated by conventional microfabrication processes involving patterning, etching and lift-off, and thus can be mass-manufactured at low-unit-cost. In comparison to the parallel plate format, the IDE platform is easier to manufacture. The IDE sensing area is often a few millimeters square and the width and separation of the electrodes on the order of a few microns. The sensitivity of this sensing platform usually increases with decreasing electrode spacing [29]. The electric field strength decays exponentially away from the electrode surface and becomes negligible after a short distance [30], and for electrodes of micron dimensions a sensing film of only a few microns thickness is therefore required [31]. Coating one side of a series of electrodes allows the whole surface of the sensing film to immediately access the environment being sensed and 
often leads to a faster response time compared to a thin film sandwiched between a pair of parallel plates. In a parallel plate configuration the upper parallel plate is often permeated with holes to allow the target molecules to reach the sensing film, but this still limits the available surface area of the sensing film. However, in the IDE format only half of the electric field lines pass through the sensing film, the rest of the electrical field passes through the lower substrate, therefore IDE sensors will have approximately half the sensitivity of an equivalent parallel plate device.

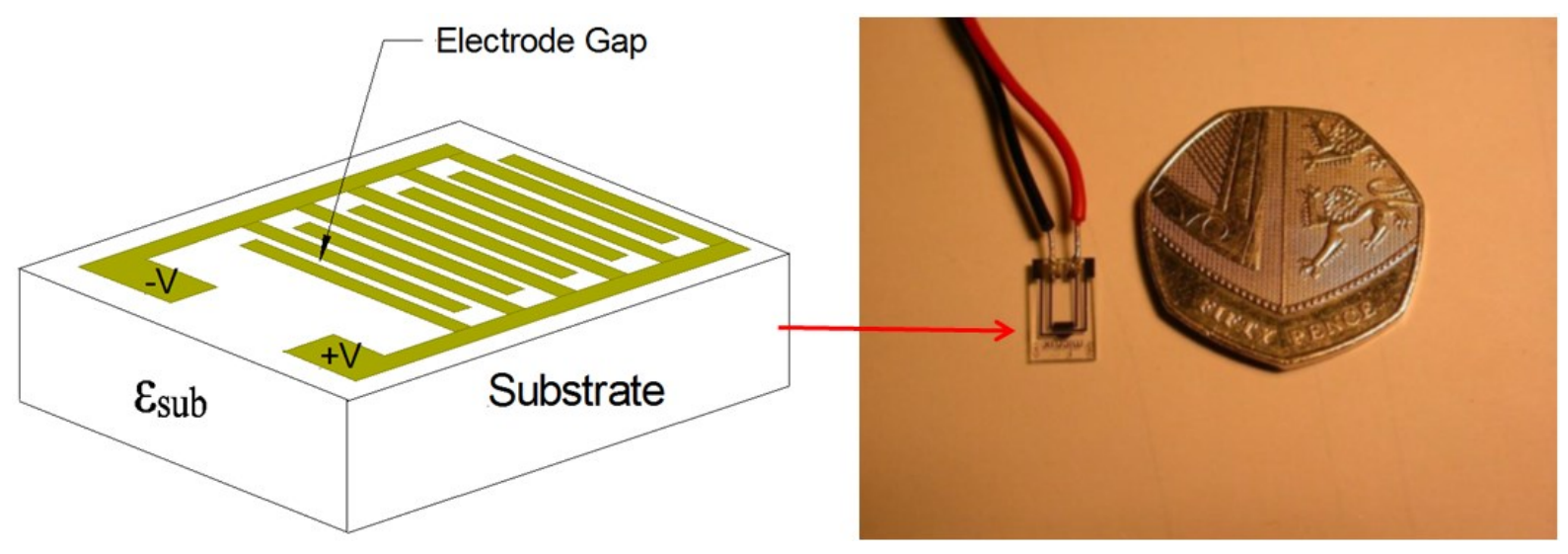

(a)

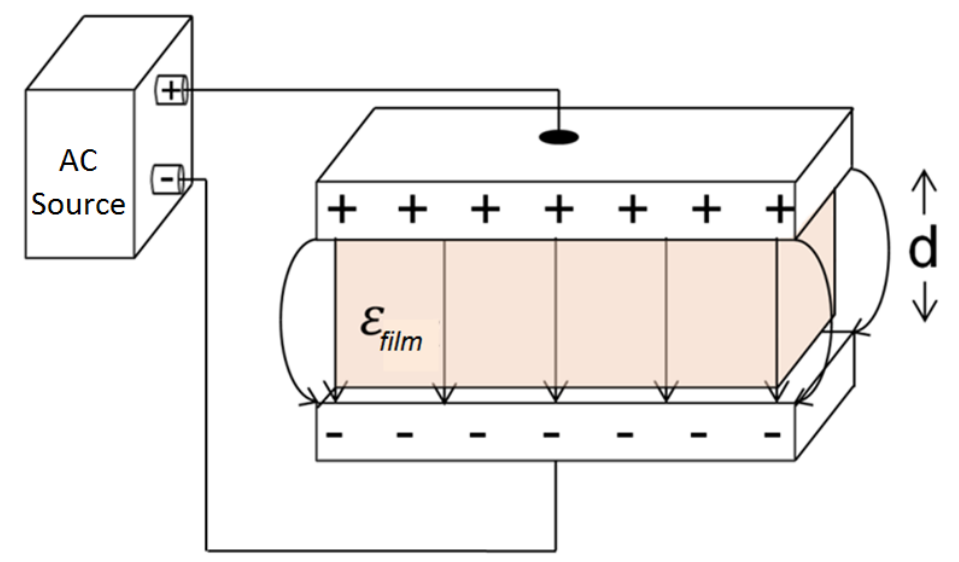

(b)

Figure 1. (a) Schematic view of interdigitated electrodes (IDEs) structure showing the general two dimensional (planar) nature, together with relative size of 15 pairs of gold IDEs on glass (photo). (b) Topology of parallel plate capacitor showing sensing film sandwiched between overlapping electrodes.

A sensor that measures capacitance changes of a chemically sensitive film is known as a chemicapacitor. Chemicapacitors can operate at room temperature and use a sensing mechanism that is ideally non-dissipative and hence requires very low-power (as low as a few $\mu$ Watts $[32,82]$ ). Multiple chemicapacitor sensors can be fabricated on a single chip with different sensing films to form a compact array of micro-sensors targeting different molecules. 


\subsection{Sensing Mechanisms of Chemicapacitor Sensors}

Igreja and Dias [33] conducted analysis on an IDE structure having one sensing layer of dielectric constant $\varepsilon_{\text {Layer }}$ and a thick substrate of dielectric constant $\varepsilon_{\text {Sub }}$ and showed that the total capacitance is given by:

$$
\text { Cap }=(N-3) \frac{C_{I}}{2}+2 \frac{C_{I} C_{E}}{C_{I}+C_{E}}
$$

where $\mathrm{N}$ is the number of electrodes and $\mathrm{C}_{\mathrm{I}}$ and $\mathrm{C}_{\mathrm{E}}$ are the half capacitance of one interior electrode and the capacitance of one outer electrode given by:

$$
\begin{aligned}
& C_{I}=\varepsilon_{0} L\left[\frac{K\left(k_{I_{-} \infty}\right)}{K\left(k_{I_{-} \infty}^{\prime}\right)}+\left(\varepsilon_{\text {Layer }}-1\right) \frac{K\left(k_{I_{-} \text {Layer }}\right)}{K\left(k_{I_{-} \text {Layer }}^{\prime}\right)}+\varepsilon_{\text {Sub }} \frac{K\left(k_{I_{-} \infty}\right)}{K\left(k_{I_{-} \infty}^{\prime}\right)}\right] \\
& C_{E}=\varepsilon_{0} L\left[\frac{K\left(k_{E_{-} \infty}\right)}{K\left(k_{E_{-} \infty}^{\prime}\right)}+\left(\varepsilon_{\text {Layer }}-1\right) \frac{K\left(k_{E_{-} \text {Layer }}\right)}{K\left(k_{E_{-} \text {Layer }}^{\prime}\right)}+\varepsilon_{\text {Sub }} \frac{K\left(k_{E_{-} \infty}\right)}{K\left(k_{E_{-} \infty}^{\prime}\right)}\right]
\end{aligned}
$$

where $\varepsilon_{0}$ is the relative permittivity of free space, $\mathrm{L}$ is the length of the electrodes, and $\mathrm{K}(.$.$) is the$ complete elliptical integral of the first kind. The first term within the square brackets represents the contribution to capacitance of an infinite air layer, the second term the sensing film, and the third term the thick substrate. From these equations it is clear that for other parameters remaining fixed, the capacitance is directly proportional to the dielectric constant of the sensing layer $\left(\varepsilon_{\text {Layer }}\right)$.

For a capacitor constructed from two parallel plates (as shown in Figure 1(b)) with the gap (d) between the plates filled with air, then if the plate area $\mathrm{A}>\mathrm{d}$, the electric field $\mathrm{E}$ can be assumed to be uniform between the plates. The capacitance $\mathrm{C}$ is defined as the electric charge stored by the capacitor to the voltage applied [34]:

$$
C=\frac{Q}{V}=\frac{\varepsilon_{0} A}{d}
$$

If a sensing film is placed between the plates the voltage between the plates will reduce and the capacitance will increase in proportion to the dielectric constant of the film $\left(\varepsilon_{\text {film }}\right)$ :

$$
C=\frac{\varepsilon_{0} \varepsilon_{f i l m} A}{d}
$$

Thus, as $\varepsilon_{\text {film }}$ or $\mathrm{d}$ changes in the presence of target molecules a direct change in capacitance occurs. The important design goal is to ensure that the analyte molecules can interact with the dielectric layer without hindrance from the parallel plate capacitor structure. 


\subsection{Analyte Interaction with a Polymer Thin Film}

Although different materials have been used to form the sensing film of a chemicapacitor, nonconducting polymers are regarded as the most advantageous to be developed for chemicapacitor sensors. The absorption of the analyte by the film is accompanied by changes in the dielectric properties and/or thickness, which alters the intrinsic capacitance of the device. This leads to a measurable change in capacitance between the electrodes. These sensors do not rely on the changes in conductivity of the material and ideally are non-dissipative in power and therefore is the favored direction to pursue in the development of low-power sensors. The polymers can also be low cost offthe-shelf and unlike other sensing films (e.g. metal oxide semiconductor) polymers function at room temperature, which again reduces the power required by the sensor for normal operation. A polymer can be readily deposited using drop-cast, inkjet, or lithography [35-36]. Polymers have also shown good linearity, good reversibility, and fast response time to absorption of analytes and different polymers can be selected for their affinity to target molecules. However, in order to achieve good reversibility, only weak interactions between the target molecule and the polymer sensing film should take place. This leads to non-specific absorption and problems of cross sensitivity to other molecules in the atmosphere. This is especially true of water molecules that have an unusually high dielectric constant (76.6) and are ever-present in the environment. This cross interference has been a major stumbling block to bringing chemicapacitors to the marketplace, but researchers are finding ways around it. For example, weak selectivity to VOCs has led to the adoption of arrays of sensors with different polymer films, and post-processing of measured data using numerical methods, such as multicomponent analysis and pattern recognition, is used to identify individual molecule concentrations in a vapor mixture [37]. In addition, separate humidity and temperature sensors can be included on the sensor chip to allow calibration for changing atmospheric conditions.

\subsection{Electronic Circuits for Measuring Sensor Capacitance}

The success of miniaturized sensors will be dependent on the cost-effective integration of the sensors with low-cost, low-power electronics that offer capabilities such as signal amplification, selfcalibration, data processing, signal conditioning, convenient readout interfaces, and wireless data transmission. To facilitate commercialization, sensors need to be miniaturized and combined with electronics using standard processing used in CMOS technology that uses established processes and is amenable to batch fabrication. Chemicapacitors are advantageous in this respect as there are no complex moving parts (as found in MEMS), thus simplifying fabrication processes. The sensing films can be deposited by inkjet technology after CMOS fabrication.

In general, electronic circuits used to measure the capacitance of a chemicapacitor can be applied equally to IDE and parallel plate formats. Chemicapacitors usually produce a small capacitance change of a few $\mathrm{pF}$ or less in response to an external analyte whilst having an inherently larger intrinsic capacitance. There are also parasitic capacitances from circuit components and environmental interference. A range of electronic circuit interfaces have been demonstrated to translate the changes in capacitance of a chemicapacitor to changes in another parameter that can undergo further signal processing. These have included simple capacitance to frequency conversion using an oscillator formed from either a timer or an op-amp. If the ubiquitous low cost 555 timer is 
configured in astable format (see Figure 2(a)) it will continuously oscillate and output a square waveform whose frequency is given by

$$
f=\frac{1.44}{\left(R_{1}+2 R_{2}\right) C_{\text {sensor }}} .
$$

Therefore a measure of the output frequency gives a measure of the instantaneous capacitance value of the sensing capacitor. Similarly, a relaxation oscillator can be constructed from a single op-amp (see Figure 2(b)). The voltage level to the inverting input of the op-amp depends on the charge on the capacitor. For a low level of charge the op-amp output will be high (approx. $+\mathrm{V}_{\mathrm{cc}}$ ) and the capacitor will charge through $\mathrm{R}_{1}$ until the voltage at the inverting input rises above the non-inverting input of the op-amp which will cause the op-amp output to switch to low (approx. $-\mathrm{V}_{\mathrm{cc}}$ ) and the capacitor will begin to discharge until the voltage at the inverting input falls below the non-inverting input and the op-amp output is switched again to high. In this way the output of the op-amp will oscillate at a frequency dependent on the RC time constant. Often a comparator, which has a faster rise and fall time, is used instead of an op-amp. A microcontroller can be used to count the output pulses from an oscillator over a fixed time period to estimate the frequency. The use of these oscillators to determine an unknown capacitance negates the need for either a comparator or an analog-to-digital converter (ADC) within the microcontroller. However, temperature changes can alter the values of the circuit components producing errors, and these simple circuits cannot exclude excess (parasitic) capacitance introduced, for example, via connection wiring linking a chemicapacitor sensor to a circuit, thus close integration of the sensor and electronics is a necessity.

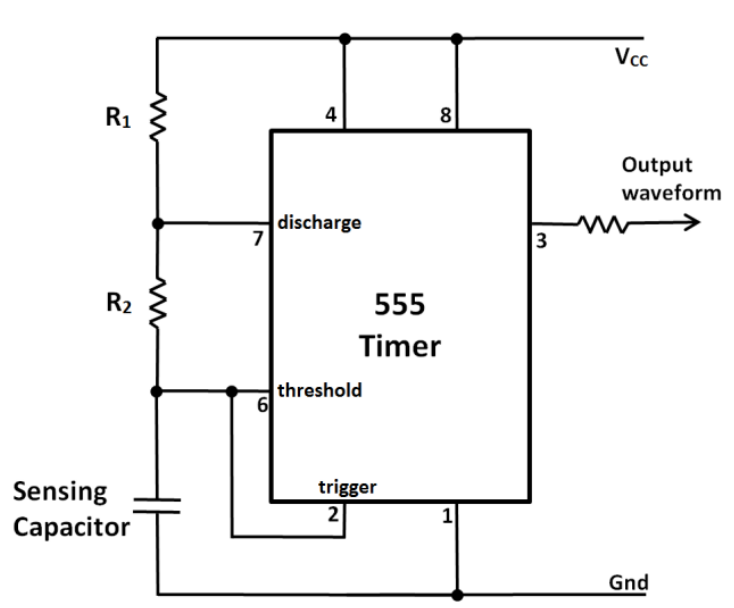

(a)

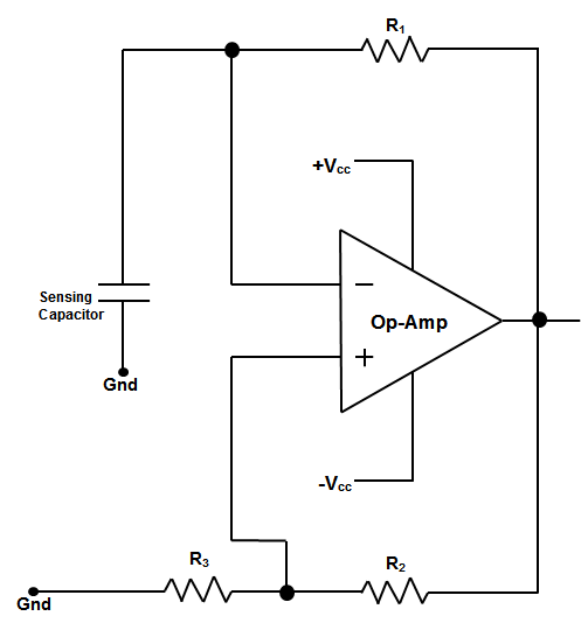

(b)

Figure 2. Schematic of capacitance-dependent oscillator circuits based on (a) a 555 timer (b) op-amp.

Direct capacitance to voltage conversion (followed by an ADC) can also be implemented by an AC signal applied to a simple capacitance divider circuit consisting of the unknown (sensing) capacitor and a fixed (usually larger) capacitor of known capacitance. As the capacitance of the sensing capacitor changes the reactance (and the voltage) across it changes and thus the output voltage from the midpoint on the two capacitors will change. The stability of this voltage output will be 
dependent on the stability of the input voltage and the fixed capacitor. For more accurate measurements a capacitance bridge circuit (formed from two capacitance dividers) can be used as this arrangement is less dependent on fluctuations in the input voltage, and the unknown capacitance can be balanced against three capacitors whose variation with temperature can be matched.

Charge transfer/balancing methods have also been used to determine the capacitance of a sensor capacitor. Figure 3 illustrates a method for transferring charge from a sensing capacitor to a larger integrating capacitor. Switch 1 is closed until the integrating capacitor is discharged and then switch 1 is opened again. Now switch 2 is closed so that the sensing capacitor is charged up to the level of the reference voltage and switch 2 is then reopened. By closing switch 3 this accumulated charge is distributed between the sensing and integrating capacitors to equalize the voltage between them. The voltage that appears across the integrating capacitor will be directly proportional to the charge on it, which was transferred from the sensing capacitor. The subsequent closing of switches 2 and 3 only in order allows the voltage on the integrating capacitor to build up to a predetermined value, while a timer records the number of charge transfer cycles (from the sensing capacitor) needed to achieve this. The converse method can also be used where the number of transfer cycles is fixed and the resultant voltage across the integrating capacitor is then read out via an ADC, and thus a varying output voltage will be in proportion to a varying capacitance of the sensing capacitor. This method, although simple in concept, requires a stable reference voltage and analog switches that have inherently smaller capacitances than that being measured.

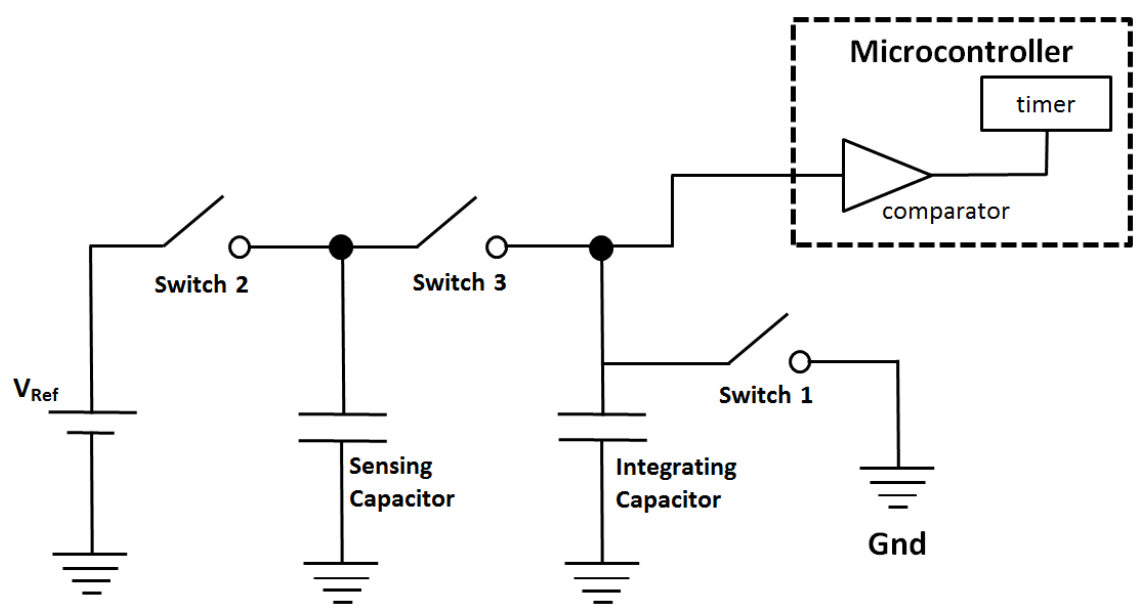

Figure 3. Capacitance measurement by charge transfer to an integrating capacitor.

In recent years direct conversion of the capacitance to a digital signal on an IC chip using $\Sigma \Delta$ ("sigma-delta") converters has gained prominence [38]. The sensing capacitor is directly connected to the virtual ground of the IC chip reducing parasitic capacitances and an in-built temperature sensor compensates for temperature drifts by on-chip calibration. The converter incorporates an on-chip excitation source and a switched capacitor network to sample the charge on the sensing capacitor and a reference capacitor. These single IC chips have come to be known as "capacitance to digital converters" (CDCs) [39]. An input analog signal is sampled and turned into pulses whose varying number (or frequency) represents the changing analog signal amplitude (known as pulse density 
modulation) and the original signal can be recovered by time averaging methods. The errors introduced by digitizing an analog signal (quantization error) from the output are fed back (via a digital to analog converter) and subtracted from the next input signal (delta operation) to improve the resolution of the quantizer and allow a coarser quantization. By oversampling the signal, quantization noise can be spread over a larger frequency range and an in-line integrator (sigma operation) can push the noise to higher frequencies and low-pass digital filtering can reduce this noise. The CDC output is directly compatible with an $\mathrm{I}^{2} \mathrm{C}$ interface. The introduction of $\Sigma \Delta \mathrm{CDCs}$ that are directly integrated with the chemicapacitor sensor has improved the accuracy during the conversion process [40]. However, this type of converter has a limited capacitance range due to modulator overload [41], the internal signal processing (oversampling and decimation filtering ("downsampling")) require relatively higher power consumption, and they can have a relatively limited data rate $(\mathrm{kHz})$. Example CDC's developed have include the AD774x series from Analog Devices Inc. which offers up to 24-bit resolution, with a $\pm 4 \mathrm{pF}$ range and a $4 \mathrm{fF}$ accuracy for less than 10 dollars a chip. It incorporates an on-chip temperature sensor with an accuracy of $\pm 2^{\circ} \mathrm{C}$, and can accommodate 1 or 2 sensor inputs. The operating power is several $\mathrm{mW}$, but variations have included the AD7150 with a power dissipation of $300 \mu \mathrm{W}$, and the AD7147 with up to 13 sensor inputs.

\section{Review of Interdigitated Chemicapacitors}

\subsection{IDE Chemicapacitor Gas Sensors}

Due to the high dielectric constant of water and its prevalence in the atmosphere, humidity sensors based on film coated IDEs were the first widespread created chemicapacitors and were developed during the late 1970's [42]. During the 1980s chemicapacitor humidity sensors [43-44] moved beyond the demonstration stage and the commercialization of these devices followed this research. Currently humidity sensors are still the most common of the chemicapacitors on the market. In the early 1990's a chemicapacitor for non-humidity sensing using planar IDEs coated with an inorganic thin film of ion-exchanged porous zeolite was reported [45]. Adsorption of a gas into the zeolite modified the dielectric constant of the material and hence its capacitance. The sensor was located within a test chamber at an elevated temperature of $55^{\circ} \mathrm{C}$ and its capacitance was monitored with a LCR-meter at a measurement frequency of $1000 \mathrm{~Hz}$. For $1 \%$ butane in air atmosphere introduced step-wise a change in capacitance of $1.2 \mathrm{pF}$ took place with a response time between 20 to 120 seconds (depending on zeolite film thickness). Lin et al. [46] deposited an organic ormosil layer upon IDEs to create a sensor for the gas sulphur dioxide $\left(\mathrm{SO}_{2}\right)$ over a wide range of concentrations from 0.5 to $6000 \mathrm{ppm}$ with a response time less than 20 seconds.

However, the successful deployment of chemicapacitor sensors for gases (and VOCs) will be driven by their ability to operate outwith the laboratory in an environment of changing humidity and temperature, both of which affect the stability of these sensors. Therefore, Mutschall and Obermeier [47] developed a chemicapacitor for the detection of $\mathrm{CO}_{2}$ using a sol-gel film consisting of a combination of propyltrimethoxysilane (PTMS) and 3-aminopropyltrimethoxysiloxane (AMO). An integrated resistive heater on the quartz substrate around the $5 \times 11 \mathrm{~mm}$ region of IDEs (electrode 
width and gap of $5 \mu \mathrm{m}$ ) was included, which made the sensors insensitive to changes in the ambient temperature. The topology of the heater (a $300 \mathrm{~nm}$ film of molybdenum) was modelled and fabricated to produce a uniform temperature distribution across the IDEs. Thermographic imaging measurements confirmed the temperature distribution across the IDEs deviated by under $4 \%$ at $75^{\circ} \mathrm{C}$. However, the dissipated power was around $200 \mathrm{~mW}$ to maintain a temperature of $70^{\circ} \mathrm{C}$. At this temperature the sensor exhibited a $0.7 \mathrm{pF}$ capacitance change measured on an impedance analyzer for a step change of $10,000 \mathrm{ppm} \mathrm{CO}_{2}$.

Endres et al. [48] reported a chemicapacitor sensor for $\mathrm{CO}_{2}$ designed with reduced humidity interference. A sol-gel film consisting of $30 \%$ PTMS and $70 \%$ AMO was employed as a $\mathrm{CO}_{2}$ sensitive layer which was deposited gold IDEs upon a glass substrate forming the chemicapacitor (see Figure 4). This platform was integrated with a resistive heating element around the sensor that thermally cycled the device area temperature. A second resistive element formed an on-chip temperature sensor located in the center of the sensor, whilst humidity was measured with a commercial capacitive humidity sensor. The inclusion of the hydrophobic PTMS within the more hydrophilic AMO was intended to reduce sensor responses due to the presence of humidity alone. However, residual water was required to drive the reversible reaction with $\mathrm{CO}_{2}$ (equation 7) and hence the sensor performance was still humidity dependent.

$$
-\mathrm{NH}_{2}+\mathrm{CO}_{2}+\mathrm{H}_{2} \mathrm{O} \leftrightarrow \mathrm{NH}_{4}^{+} \mathrm{HCO}_{3-}
$$

In addition, to facilitate this reaction (and thus reduce the response time) required a platform temperature around $60^{\circ} \mathrm{C}$ which dissipated over $300 \mathrm{~mW}$. Calibration for changes in humidity was achieved through an iterative numerical algorithm established from step changes in $\mathrm{CO}_{2}$ and humidity. This calibration allowed almost total suppression of humidity interference and the sensor could operate reliably in room air with a resolution of $10 \mathrm{ppm} \mathrm{CO}_{2}$, with the highest resolution over the range 100 and $3000 \mathrm{ppm} \mathrm{CO}_{2}$. The sensor was successfully tested over an 8 month period within an office environment for monitoring air quality. 


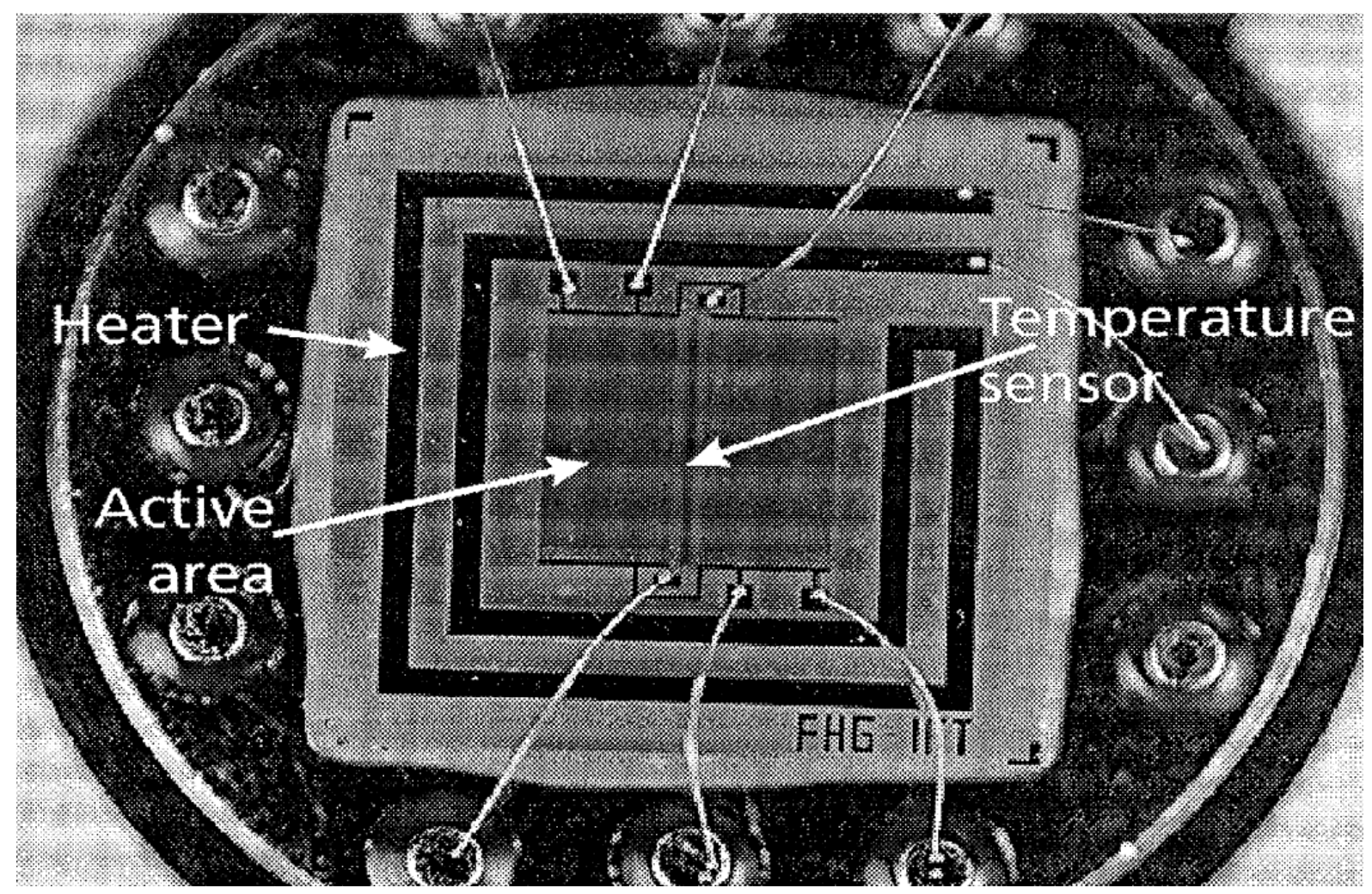

Figure 4. Early IDE chemicapacitor $\mathrm{CO}_{2}$ gas sensor incorporating on-chip temperature sensor and heater. Reproduced with permission from Endres et al. [48]. Copyright Elsevier 1999.

Additionally, in 2006, Kebabian and Freedman [49] reported the combination of a fluoropolymer (Teflon AF 2400 ${ }^{\mathrm{TM}}$ ) coated chemicapacitor integrated with a custom made capacitance balancing measurement circuit on a PCB. The specific application was the development of a lightweight and low-powered sensor for a balloon-borne platform to measure atmospheric $\mathrm{CO}_{2}$. However, water vapor was found to be an interferent producing larger signal change than for equivalent concentrations of $\mathrm{CO}_{2}$ and increasing the response time. This was exacerbated by having no on-chip reference sensor to help subtract out these effects. In a dried gas stream the response time for 0 to $100 \% \mathrm{CO}_{2}$ change was under 10 seconds with good linearity and an accuracy of between 5 and $10 \mathrm{ppm}$.

\subsection{IDE Chemicapacitor VOC Sensors}

By the mid-1990s researchers had also expanded the range of target molecules to VOCs in the atmosphere with the more widespread adoption of polymer coatings. Josse et al. [50] demonstrated chemicapacitors with 28 pairs of aluminium electrodes on glass with a range of polymer coatings for target VOCs ethanol and tetrachloroethene, whilst Casalini et al. [51] used a chemicapacitor coated with a modified polysiloxane film to reversibly detect vapors of benzene, ethanol and acetonitrile, with a response time up to 10 minutes. These early VOC (and gas) chemicapacitor sensors demonstrated during the 1990s were almost exclusively interrogated with bulk impedance analysers or LCR-meters. However, at this time Cornila et al. [52] investigated the use of established CMOS fabrication techniques to form a chemicapacitor integrated with a custom-made $\Sigma \Delta$ modulator circuit. The basic chemicapacitor platform was fabricated with the $3 \mu \mathrm{m}$ self-aligned contact CMOS 
technology and consisted on a polymer sensing film upon 32 aluminum electrodes with a $3 \mu \mathrm{m}$ gap between the electrodes. Differential measurements between a sensing and a silicon nitride passivated reference capacitor with calibration, offset and analog to digital conversion was achieved using a second-order $\Sigma \Delta$ modulation technique. The performance of four different polymers coated on the chemicapacitor was evaluated in detecting changing concentrations of a range of polar (such as ethanol, trichloromethane and propan-1-ol) and non-polar (such as tetrachloroethene, toluene, and noctane.) VOCs. For all polymers and VOCs, the chemicapacitor demonstrated good linearity with vapor concentrations from around $100 \mathrm{ppm}$ up to several thousand ppm. Also, it was found for polyepichlorohydrine (PECH) and polyetherurethane (PEUT) sensing films that their response to water vapor was less than to the target VOCs. However, the response time of these sensors was over 400 seconds and humidity effects would cause significant baseline drifts after several hours of measurement.

This system was further developed into a hybrid platform incorporating additional calorimetric and mass sensors [53]. Experiments revealed that for the IDE chemicapactor coated with a polymer layer of thickness larger than half the periodicity of the electrodes, then for a target analyte with a dielectric constant less than that of the polymer the resultant capacitance would decrease, and for an analyte dielectric constant larger than the polymer the capacitance would increase. For a layer thickness less than half the periodicity of the electrodes, the capacitance would always increase regardless of analyte dielectric constant. This consistent behavior supported earlier simulations by these authors [54] and suggested a criterion for discriminating between different analytes. The authors reported a detection limit of $5 \mathrm{ppm}$ for ethanol and $8 \mathrm{ppm}$ for toluene vapors for the chemicapacitor elements of the microsystem, with a response time for the sensor of a few seconds. These authors went on to create a battery operated handheld system incorporating six chips each with the three sensor types on them [55-56]. In this prototype device each chemicapacitor was formed from 128 IDEs with a coating of PEUT, and an on-board microprocessor conditioned the sensor signal before displaying it on a LCD display (see Figure 5). A detection limit in the range 1 to $5 \mathrm{ppm}$ for ethanol and toluene was achieved. It was noted that, as always, water vapor can cause unwanted signal changes but with the use of non-polar sensing polymers this problem can be significantly reduced. 


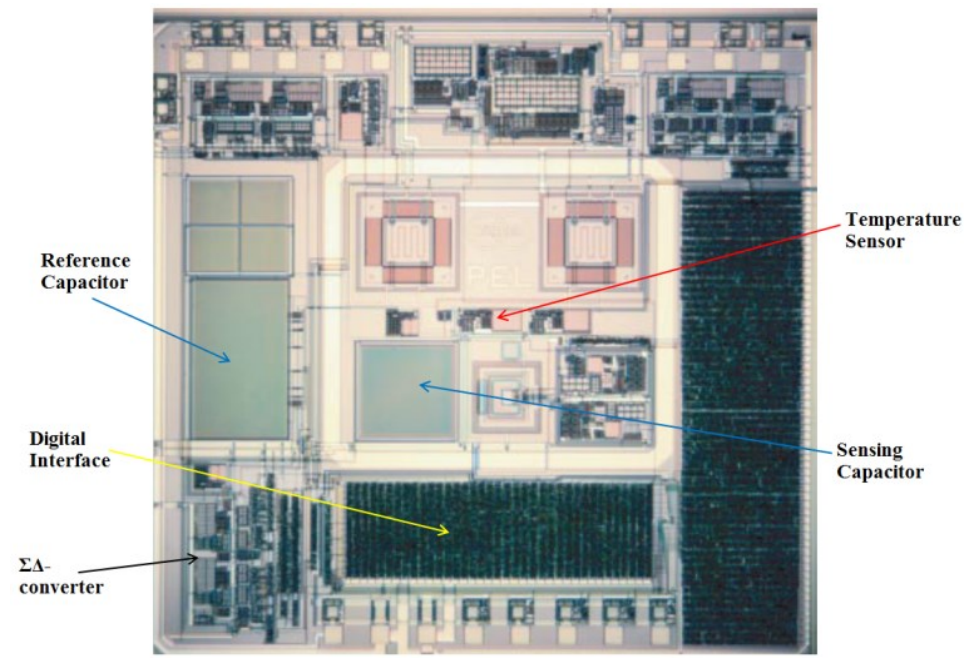

(a)

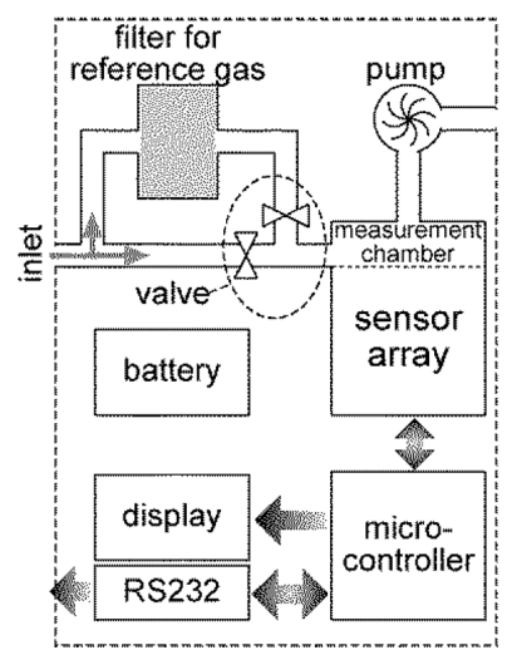

(b)

Figure 5. (a) Micrograph of system chip incorporating chemicapacitor sensors for the detection of VOCs. Reproduced with permission from Hagleitner et al. [55]. Copyright (C) Nature Publishing Group 2001. (b) Schematic of battery-powered miniaturized gas flow system, sensor array and microcontroller. Reproduced with permission from Hagleitner et al. [56]. Copyright @ IEEE 2002.

To increase the selectivity of chemicapacitors to target molecules, Igreja and Dias [57] studied the partition coefficient of polymers as a method for selecting an appropriate polymer to target a specific organic vapor within a mixture of vapors. By studying the change in capacitance over time, separate relaxation processes could be identified indicating different polymer affinity for different vapors. In addition, by modelling gold electrodes on borosilicate glass they concluded that for polymer thicknesses beyond half the sensor wavelength (equal to electrode width plus electrode separation) the sensor was not sensitive to permittivity changes in the sensing film beyond this thickness. Hence, the smaller the IDE spatial geometry that can be fabricated the thinner the sensing film can be for full utilization of the sensing geometry of the film. Subsequently, in a follow up paper, the authors reported for the first time the evaluation of a chemicapacitor array, and they combined this platform with the use of Principal Component Analysis (PCA) for vapor recognition within a mixture of VOCs in approximately 5 seconds [58]. PCA is a mathematical method that models the variation in the data to find significant patterns (correlation) within the data to create a reduced number of principal components. The number of sensors within an array will give a number of variable responses to a vapor mixture and analysis will allow clustering of the output data. The chemicapacitor resolution was $0.1 \mathrm{fF}$ and it was possible to discriminate individual vapors within the mix of toluene, n-octane, 1-propanol and ethyl acetate.

Kitsara et al. [59] began the development of an array of chemicapacitor sensors for VOCs that would be not only integrated with low-power electronics but would offer better immunity to water vapor interference. In an early version, a single quartz platform with four chemicapacitors (see Figure 6) with 400 aluminum electrodes (width and spacing of $5 \mu \mathrm{m}$ ) coated with different off-the-shelf polymer coatings was applied to discriminate target vapors within a mixture of vapors. The developed sensors were exposed to four target molecules: water, ethanol, methanol and ethyl acetate [60]. All the 
polymers were found to exhibit a sensitivity (measured on a capacitance meter) to each of these targets whose magnitude was dependent on the dielectric constant of the molecule and the absorptive capacity of the polymer. The ratio of these differential sensitivities gave a method to selectively discriminate a binary mixture of these vapors. It was also shown consistently that the magnitude to the sensor response to a binary mixture was the sum of the responses to the individual pure analytes, thus allowing identification of the individual analyte within the mixture. The response was found to be linear and reversible up to concentrations of $4000 \mathrm{ppm}$ of all the analytes. PCA was applied to enable further discrimination between vapors consisting of $5000 \mathrm{ppm}$ of individual target molecules and binary mixtures of these. Figure 7 shows the ability of this analytical method to isolate the individual targets and their binary mixtures. Subsequently, the same authors found that reducing the IDE critical dimensions (electrode width from 5 to $2 \mu \mathrm{m}$ ) did not affect the sensor performance [61], and thus does not impede increased miniaturization. Following the work of Igreja and Dias [57] this could also allow thinner sensing films. This same research group went on to combine an eight sensor array with low a power data readout module consisting of a 24-bit CDC (AD7746) and USB connection to a computer with developed user interface software [62]. The developed electronics introduced an estimated $0.2 \mathrm{fF}$ of noise. The in-built temperature sensor within the CDC compensated for sensor drift due to temperatures changes. A demonstration of a detection limit of $90 \mathrm{ppm}$ for toluene and 30 ppm for p-xylene was achieved using this small low-powered, low-cost chemicapacitor system (see Figure 8). In a further study [63] the authors found that with the use of more hydrophobic polymers, the detection of low concentrations of target VOCs was possible in the presence of normal levels of humidity (up to $50 \% \mathrm{RH}$ ). For a chemicapacitor coated with the hydrophobic polymer poly(ethyl methacrylate) the limit of detection (LOD) for xylene, toluene, ethyl acetate was 19, 13 and $76 \mathrm{ppm}$ respectively. The developed chemicapacitor system integrated with the single chip CDC outperformed a bulk commercial capacitance meter (HP4278A) for the detection of four VOCs in terms of LOD and sensitivity. This 8-chemicapacitor array system was applied to monitoring alcoholic fermentation of grape wine as an alternative to offline GC/MS methods [64]. By using this array of sensors the production of alcohol could be measured and the discrimination between normal and spoiled fermentation could be achieved through the sensing of by-products such as ethyl acetate and acetic acid. 


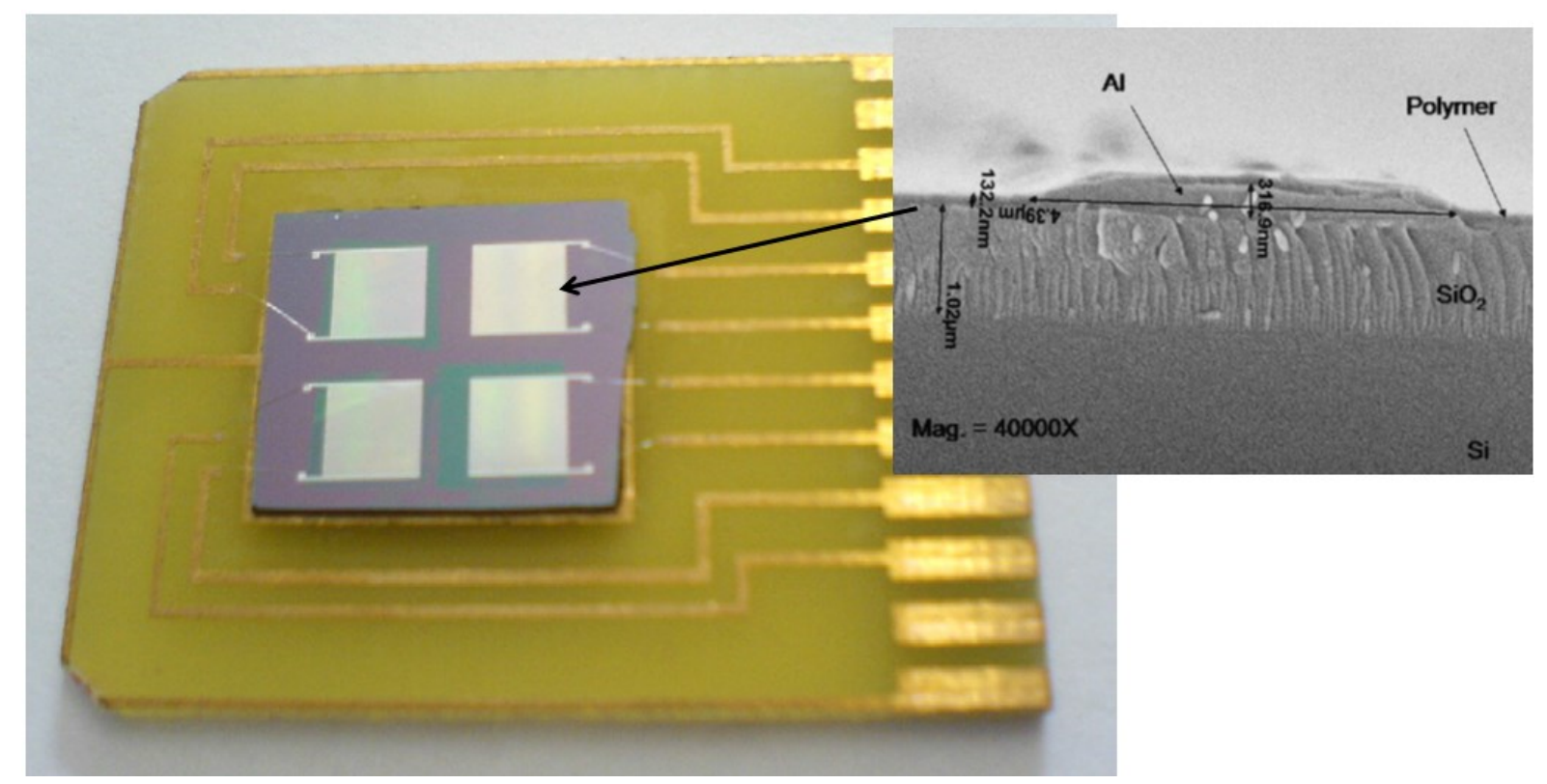

Figure 6. Four chemicapacitor sensors fabricated as aluminum IDEs on a quartz substrate (inset) attached to a PCB. Reproduced with permission from Kitsara et al. [59]. Copyright Elsevier 2007.

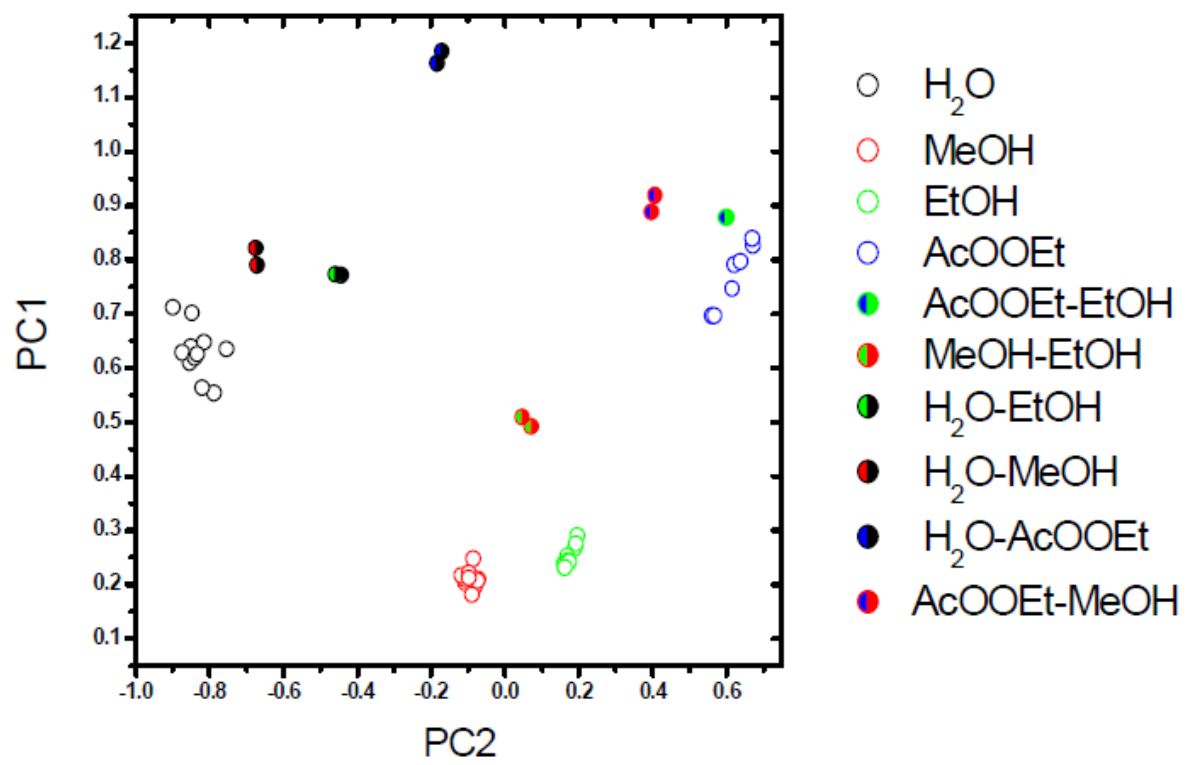

Figure 7. Identification of individual molecules and binary mixtures using principal component analysis (PCA) on the data collected from a chemicapacitor array of four sensors. Reproduced with permission from Manoli et al. [60]. Copyright (C) IEEE 2008. 


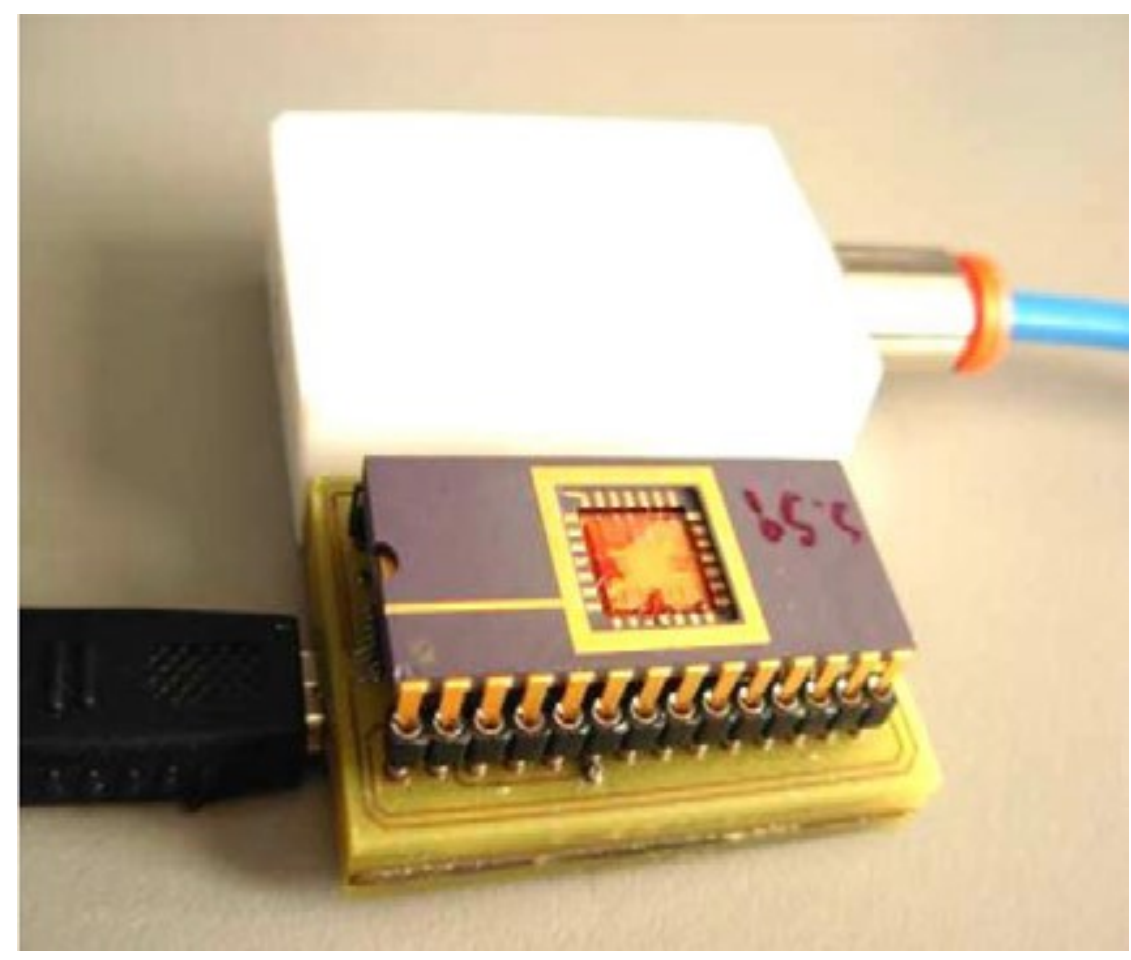

Figure 8. Compact $\left(10 \mathrm{~cm}^{2}\right)$ electronic vapor sensing module incorporating eight chemicapacitor sensors powered via a computer USB connection. Reproduced with permission from Botsialas et al. [63]. Copyright Elsevier 2013.

Emadi et al. [65] simulated and fabricated a novel $50 \mu \mathrm{m}$ thick silicon cantilever structure containing a chemicapacitor with a resistive ITO heater adjacent to the copper IDEs to maintain the sensor temperature to within $1{ }^{\circ} \mathrm{C}$ across the platform to reduce sensor drifts with environmental temperature fluctuations. This particular chemicapacitor was aimed towards grain storage monitoring where significant temperature variations are encountered. The sensing film was a layer of poly(vinyl acetate) drop cast onto the IDEs up to a thickness of $8 \mu \mathrm{m}$. In the absence of moisture, the sensor demonstrated a near linear and reversible detection of 1-octanol, a VOC released when stored grain spoils. Subsequently, the same authors [66] went on to use a poly (N-vinylpyrrolidone) coated chemicapacitor together with frequency spectroscopy to demonstrate a differential sensor response to water and 1 octanol vapors at different frequencies. The authors suggest that employing frequency spectroscopy (together with temperature modulation) offers a means to discriminate between octanol and water using a sensor array. The developed chemicapacitor was shown to detect 1 octanol to ppm levels. However, the time for this sensor to reach equilibrium after step changes in 1 octanol was around 15 minutes.

Detection of explosives is a priority to increase security in society and to protect infrastructure. Detection of explosives by detecting their vapor is currently undertaken using sniffer dogs (which require training and a handler) or the deployment of developed technology (such as ion mobility and optical spectrometry, x-ray dispersion, terahertz imaging), which is currently bulky and of high-cost, and again requires personnel to operate the equipment. Explosives sensors have been reported that are based on fluorescence sensing, for example, ICX Technologies Inc. Overall, such optical sensors are complex and expensive systems, requiring the integration of light sources, optical filters, sensitive 
photodetectors and electronics [67]. Biosensors can be used for explosives detection and the majority of these deals with liquid-mediated formats and optical readout [68]. These techniques are however generally not reversible, and thus the sensor is not reusable. Chemicapacitors offer a viable alternative to these technologies.

In 2005 Patel et al. [69] fabricated 16 IDE capacitors on a single chip each with 25 pairs of electrodes elevated above a substrate using $2 \mu \mathrm{m}$ tall posts (see Figure 9). These particular electrodes were fabricated using micromachining of conductive polycrystalline silicon fabricated using a MultiUser MEMS Process (MUMPs) process. Each sensor was coated with a different polymer using inkjet techniques. These polymers were selected for their particular affinity for target vapor molecules. The sensors were integrated with a custom electronic circuit incorporating a charge-to-voltage converter (driven by a $10 \mathrm{kHz}$ charge/discharge drive voltage) followed by a sample-and-hold amplifier. The timing and sampling were controlled by an on-board microcontroller that output an analog voltage (proportional to the sensor capacitance) to a RS232 interface. The circuit was hermetically sealed, whilst the sensor was left exposed to the atmosphere (except for a protective screen to reduce dust ingress). A reference sensor on the chip with no polymer provided a subtractive signal to reduce noise. It was demonstrated that the sensor showed a high sensitivity to the explosive compound 2, 6DNT to less than $10 \mathrm{ppb}$ in a stable $60 \%$ relative humidity. However, variations in humidity would cause corresponding fluctuations in the detected signal as water vapor was a competing analyte, and an increasing environmental temperature reduced the LOD. In a later analysis the response time of these elevated IDE structures was measured as under $100 \mathrm{~ms}$ for absorption and 2 seconds for desorption of an analyte [87].

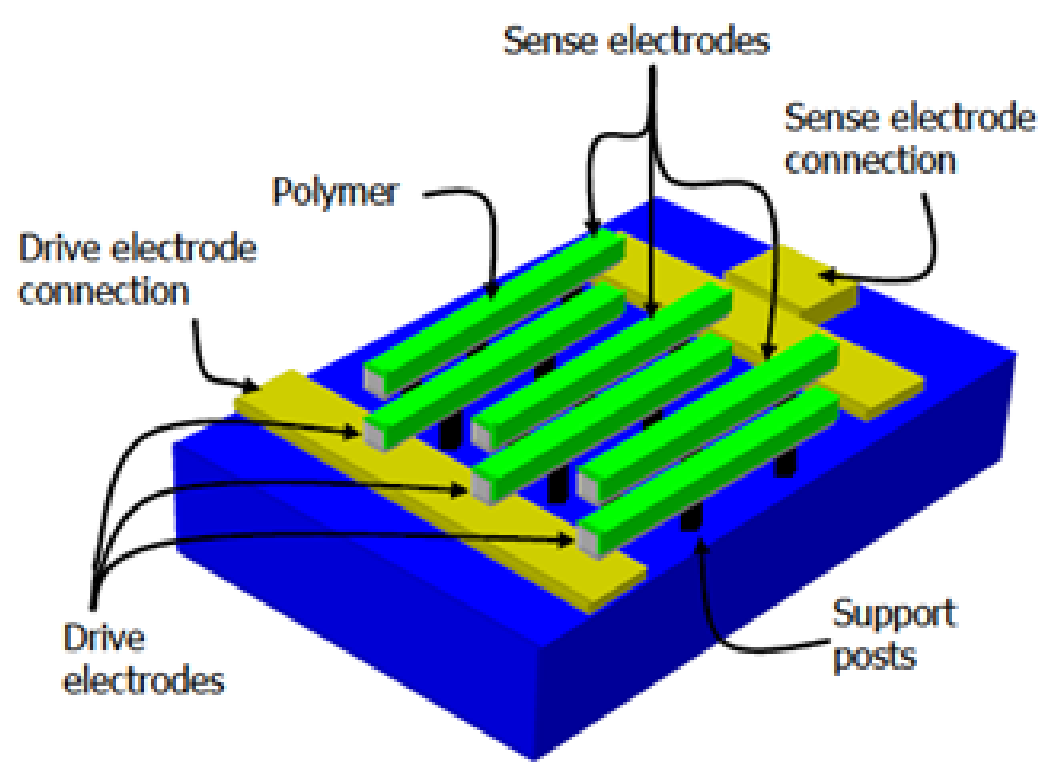

Figure 9. Schematic of three dimensional micromachined IDEs platform subsequently coated with a sensing polymer to form a chemicapacitor. Reproduced with permission from Patel et al. [69]. Copyright 2005 Society of Photo Optical Instrumentation Engineers.

Vobecka et al. [70] investigated an alternative method of sensor polymer fabrication and deposition in comparison to established techniques such as inkjet printing [36] or spin-coating 
followed by lithographic patterning [35]. These polymers were designed to specifically target chemical vapors containing the nitro $\left(\mathrm{NO}_{2}\right)$ group (which is found in high explosives). The overall aim was to minimize the cost of the final sensors. Firstly, rather than use off-the-shelf polymers, which have cross-sensitivities to a range of VOCs and thus require an array of sensors (as well as numerical algorithms), bespoke nitro-group sensitive monomers were chemically synthesized that could be deposited as a polymer by electrochemistry. Electrochemical polymerization is an inexpensive, high-yield, mass-producible deposition technology that enables controlled localized growth (on the scale of nanometers) of a polymer from a monomer solution onto IDEs by passing a small current through the electrodes for approximately 40 minutes. This has the potential of achieving a single step process that can be employed to selectively fabricate multiple sensors on a single silicon or glass substrate by controlling the flow of current. In comparison, spin-coated and plasma etching has the disadvantage of requiring expensive microfabrication equipment, whilst ink-jet printing is not compatible with producing small structures of just a few microns size - ink-jet systems yield droplets that are tens of microns in size [71]. Both of these technologies also require that the polymers are soluble in common organic solvents, whereas electrochemical deposition is not limited by this. In addition, the ability to chemically tailor the monomer allows the resultant polymer to be adapted to new targets such as the explosive precursor materials ammonia and formaldehyde which are gaining importance within the security community.

Br-ProDOT (3,4-[2(p-bromophenyl)propylene] dioxythiophene) monomers were synthesized (see Figure 10(a)) and electrochemically grown onto gold IDEs, each consisting of 25 pairs of electrodes with a spacing of $15 \mu \mathrm{m}$ (see Figure 10(b)). On a single sensor substrate a Br-ProDOT film could be grown on one IDE (nitro sensor) and a poly(3,4-ethylenedioxythiophene) (PEDOT) film on the neighboring IDE to act as a reference sensor to subtract signal changes due to any humidity variation.
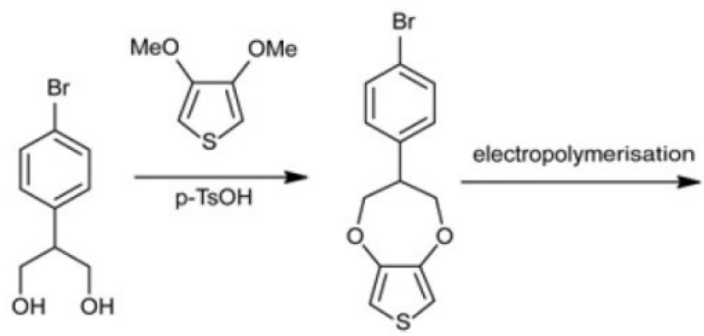

(a)

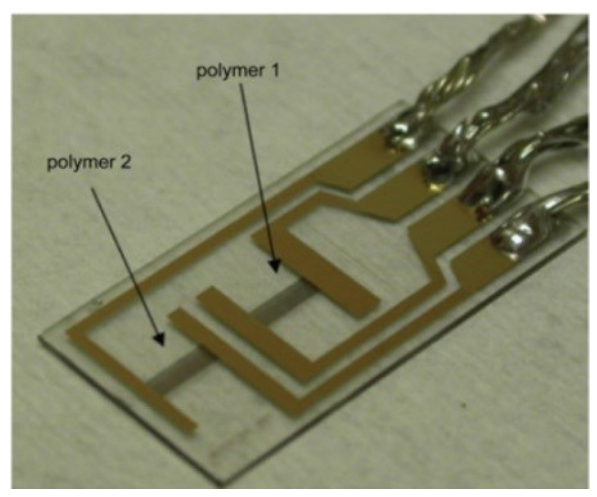

(b)

Figure 10. (a) Chemical synthesis of brominated-ProDOT and its corresponding polymer. (b) Glass substrate with two IDE devices individually coated with polymer films using electropolymerization.

These sensors were placed into a test chamber and exposed to controlled vapors of nitrobenzene, 2-nitrotoluene, and a range of VOCs commonly found in the atmosphere [72]. The interaction with the nitro group affected the conformation of the polymer and induced charge transfer between the film 
and the $\mathrm{NO}_{2}$ containing molecule. Figure 11 demonstrates the higher affinity for nitro-bearing compounds leading to a capacitance response that was up to 3 orders of magnitude higher than other vapors that do not contain the $\mathrm{NO}_{2}$ groups. The initial sensors demonstrated a rapid, linear and reversible response to around $20 \mathrm{ppm}$ (limited by the available test vapors). For the detection of explosives sub-ppm detectable concentrations are desirable, and therefore the authors altered the synthesis chemistry of the monomer to create a polymer incorporating spiro compounds to greatly increase the porosity of the polymers [73]. The resultant chemicapacitors exhibited a selective and reversible detection of nitrotoluene and nitrobenzene vapors to a concentration of around $200 \mathrm{ppb}$ in room air (see Figure 12). It should be noted that these prototype sensors were not integrated with onchip readout electronics, which could further reduce parasitic noise levels and achieve lower detection limits.

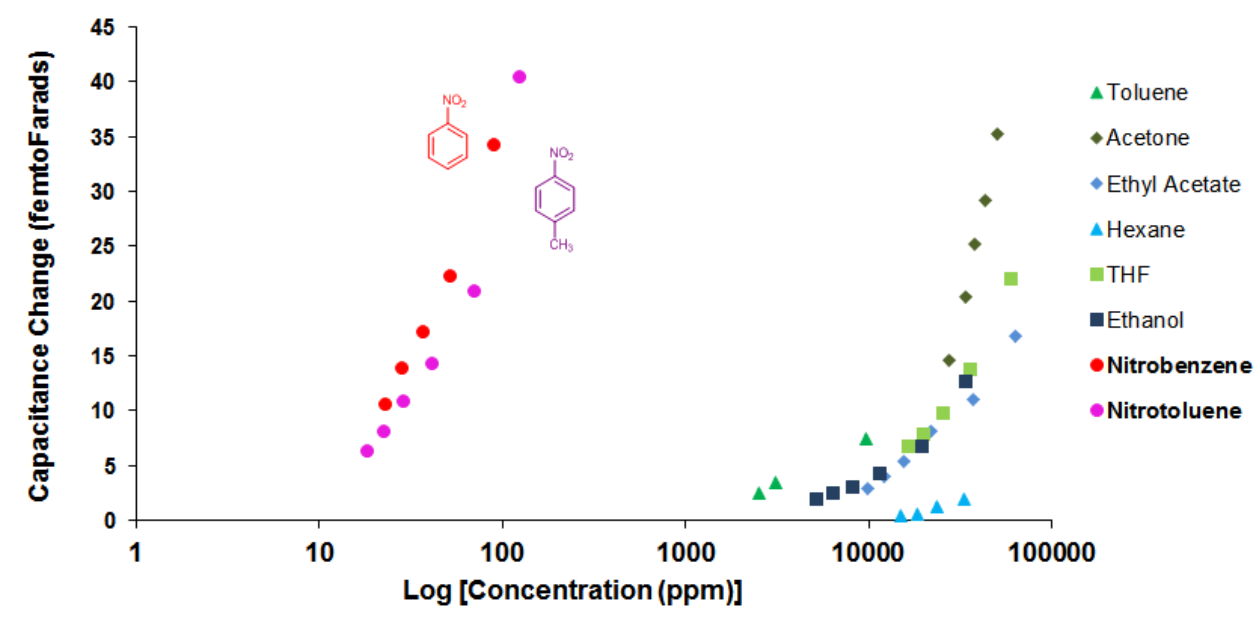

Figure 11. Measured capacitance change of a Br-ProDOT film (average thickness $400 \mathrm{~nm}$ ) for changing concentrations of several common VOCs shown in relation to nitroaromatic compounds.

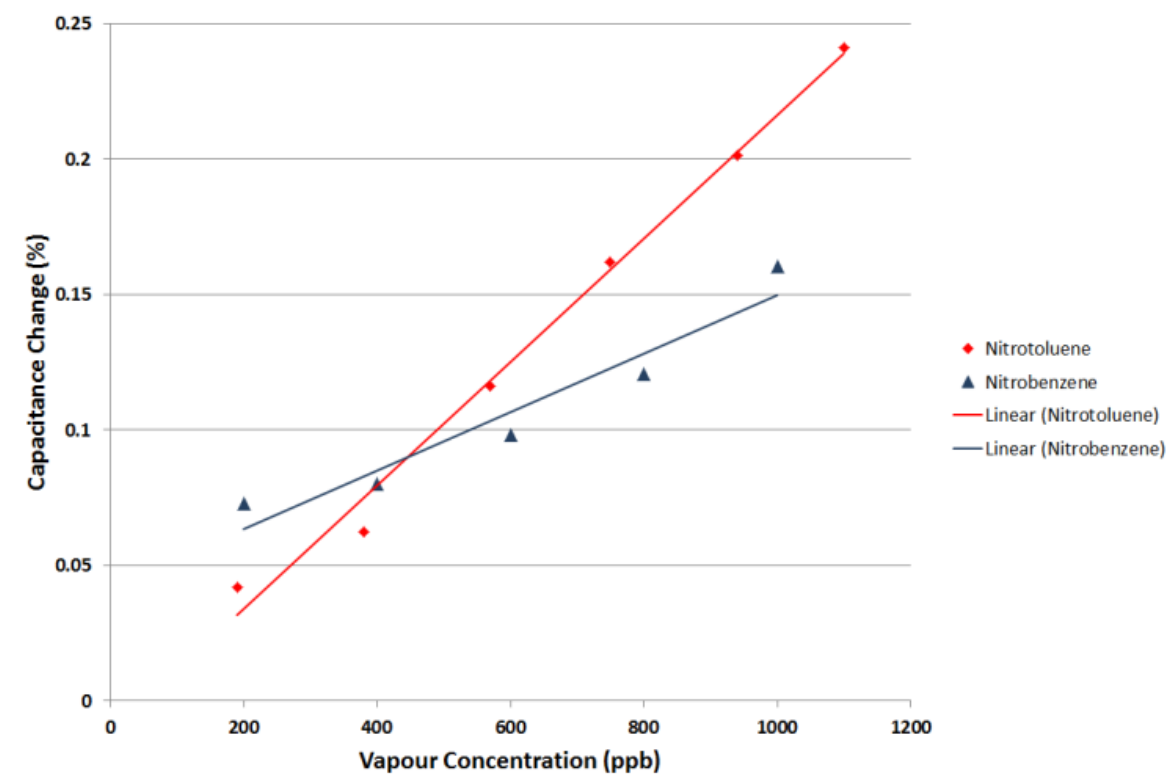

Figure 12. Linear response of a Br-ProDOT:Spiro coated chemicapacitor to nitrotoluene and nitrobenzene vapors down to $200 \mathrm{ppb}$ in room air. 


\subsection{Wireless interrogation of IDE Chemicapacitors}

With the establishment of the chemicapacitor as a viable miniature sensing platform for a wide variety of target VOCs (and gases), researchers have also turned to demonstrating that these sensor platforms can be utilized with wireless technology as a means to increase the applications, to further reduce the basic cost of these devices, and increase the autonomy of these systems. Radio-frequency identification (RFID) is a wireless technology used for communicating stored data from small devices (tags) attached to, or sealed within, containers. A standard passive tag is a low cost (usually $<\$ 1$ ) microchip with an antenna that draws power from a tag reader by electromagnetic induction and transmits data back to the reader. At present these passive tags have simple functionality. For more complex functions such as sensing, a battery can be integrated with the tag; however, a battery has a limited lifetime. More recently, adding energy harvesting [74] or energy scavenging from WiFi signals [75] capabilities to these tags have been investigated. Since capacitive sensing is a nondissipative method, a combination of such technology with miniaturized sensors has obvious applications and benefits for collecting information on the condition of the environment around and within the item of interest. Essentially, this could form the basis of intelligent labels during storage and transportation of goods, or environmental monitoring by fixed or wearable systems. However, such sensing devices must consume a minimum of power.

In an early example of a passive sensor in 2001 Ong and Grimes [76] used a non-polymer sensing film on a chemicapacitor to demonstrate wireless interrogation. The IDEs connection lines were extended to form a square spiral inductor and thus create an inductor-capacitor resonant circuit (see Figure 13(a)). The authors reported the deposition of a $200 \mu \mathrm{m}$ thick layer film comprising a composite of carbon nanotubes (CNTs) and $\mathrm{SiO}_{2}$ onto a further $150 \mu \mathrm{m}$ insulating layer of pure $\mathrm{SiO}_{2}$ was deposited upon the IDE capacitor of the sensor. The sensor did not have its own power supply. This sensor was placed inside a sealed chamber and interrogated with an antenna loop situated $15 \mathrm{~cm}$ from the chamber (see Figure 13(b)) which was linked to an impedance analyzer to supply electromagnetic energy. The sensor has a resonant frequency that corresponds to the highest absorbed power by the spiral inductor and thus changes the normal impedance spectrum of the antenna loop. The resonant frequency is given by [77]

$$
f=\frac{1}{2 \pi \sqrt{L C}}
$$

where $\mathrm{L}$ is the mutual inductance and $\mathrm{C}$ is the capacitance. Thus the value of resonant frequency changes as the effective complex permittivity of the coated $\mathrm{CNT}-\mathrm{SiO}_{2}$ layer varies in response to the surrounding $\mathrm{CO}_{2}$ concentration being altered. The impedance of the loop antenna itself was removed by measuring the impedance spectrum without the sensor in place. In dry air the response time of the sensor for a step change from 0 to $100 \% \mathrm{CO}_{2}$ was approximately 45 seconds, but in the presence of humidity there was a strong attraction between the CNTs and water vapor which increased the response time to tens of minutes (which was dependent on the environmental temperature). Therefore, 
the $\mathrm{CO}_{2}$ sensor was investigated in conjunction with a $\mathrm{SiO}_{2}$ only coated sensor and an uncoated sensor to calibrate for the effects of temperature and humidity, and it was demonstrated that this configuration could produce a linear sensor for up to $25 \% \mathrm{CO}_{2}$ to within $\pm 3 \%$. The passive nature of this sensor lends itself to unattended monitoring of the environment over extended periods of time or within sealed packaging containing medicines or food [78].

More recently, Chen et al. [79] investigated the creation of low-cost polymer-coated chemicapacitor sensor combined with a RF resonant sensor that can be developed for use with a RFID reader. The authors interrogated a resonant sensor with an RF wave whose time delay and amplitude were modified by the chemicapacitor response to changing concentrations of ethanol vapor. By monitoring both these parameters, interrogation by frequency sweeping can be avoided, which allows easier integration with existing passive RFID technology. The chemicapacitor was coated with polymers OV-225 and OV-275, and the detection resolution was around 1000 ppm with a response time of 2 minutes for this prototype wireless passive sensor. As well as industrial or food packaging monitoring, such a sensor could have biosensing applications to measure alcohol gaseous vapors emitted from the human skin as a measure of individual intoxication. 


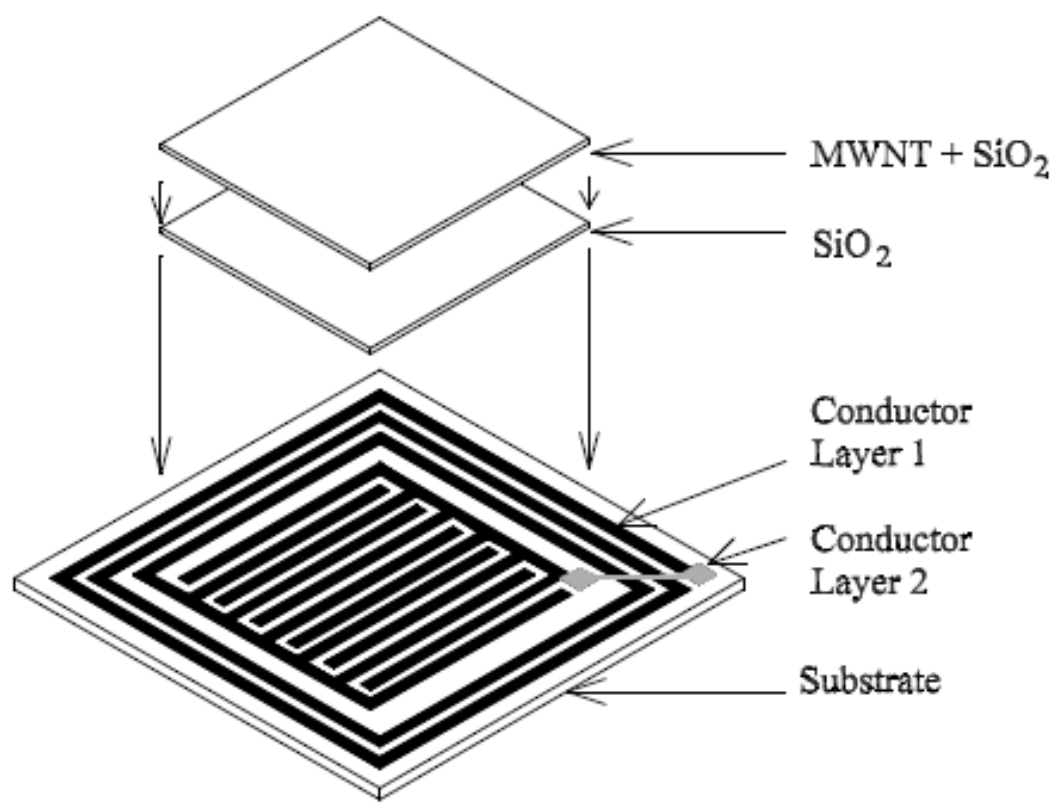

(a)

Dry air

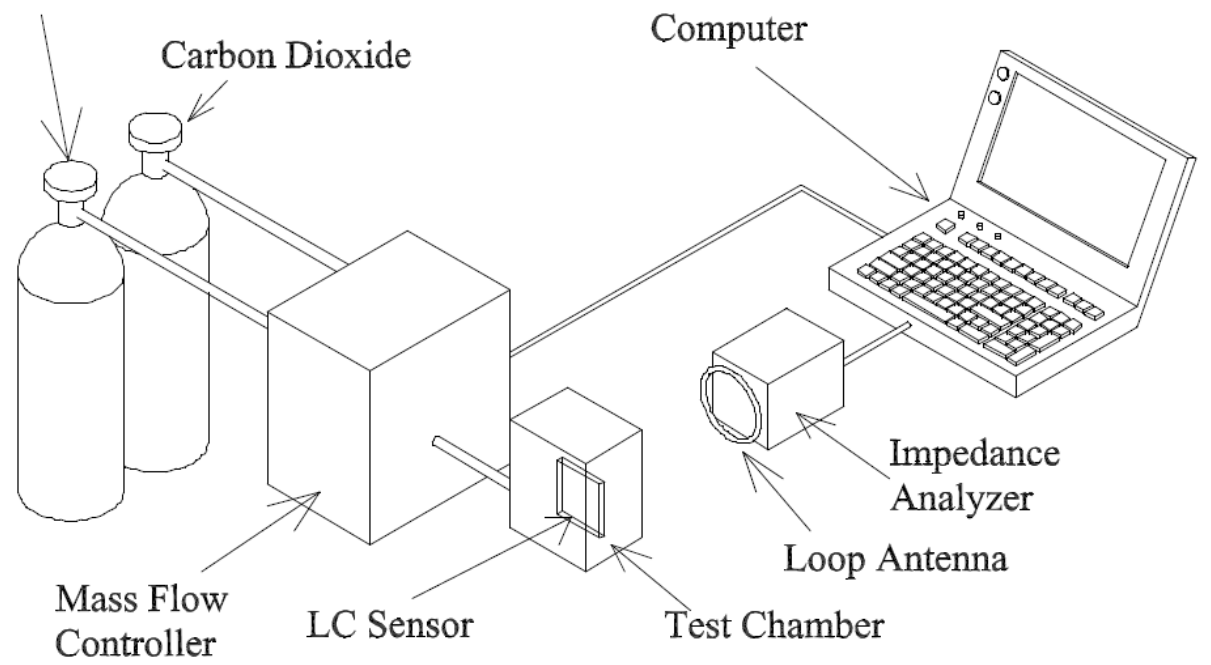

(b)

Figure 13. (a) Chemicapacitor IDEs patterned to form an inductor-capacitor resonant circuit coated with a multi-walled carbon nanotube (MWNT)- $\mathrm{SiO}_{2}$ composite. (b) Remotely interrogating the passive chemicapacitor $\mathrm{CO}_{2}$ sensor inside a sealed chamber using impedance spectroscopy. Reproduced with permission from Ong and Grimes [76]. Copyright MDPI AG 2001.

\subsection{IDE Chemicapacitor Sensors on Flexible Substrates}

Traditionally glass or silicon has been used as the inert substrates for IDEs. Plastic substrates offer the advantages of light weight and low-cost manufacture, as well as a flexibility that could allow the sensor to follow the contours of a surface (e.g. the human body). Oprea et al. [80] investigated temperature, gas and humidity resistive/capacitive sensors on plastic substrates. Two IDE structures were formed on polyimide foils, one coated with a sensing polymer ( $30 \mu \mathrm{m}$ thick of either PEUT or PDMS) and the other was left blank as a reference sensor, together with a temperature sensor formed 
from a serpentine platinum wire (see Figure 14(a)). These sensors were directly integrated with a differential $\Sigma \Delta$ CDC (AD7746) for low-power signal readout (10 $\mu \mathrm{W}$ on stand-by) and the data was sent through an $\mathrm{I}^{2} \mathrm{C}$ bus to a computer for analysis (see Figure 14(b)). Several numerical algorithms were implemented. The polyimide substrate is sensitive to humidity changes in the ambient atmosphere but this also offers the substrate itself to be used as a humidity sensing element for numerical elimination of this interferent. Experiments revealed that both the sensing and reference sensors had a significant responses to humidity changes, and to reduce this unwanted sensor drift the signal from the reference channel was subtracted from that of the sensing channel, which also helped reduced the response time. Changes in humidity would, however, still cause signal changes from the sensing layer itself and therefore a second numerical operation was implemented whereby the sensing layer drifts due to humidity was subtracted from the overall sensing layer signal. Using sensing and reference sensors also helped to reduce electrical noise which was then further reduced by the in-built features of the differential CDC. With this calibration methodology the researchers were able to estimate the overall resolution of the sensing system at $10 \mathrm{aF}$. Target vapors of n-hexane, n-propanol, ethanol, toluene and ammonia were investigated under 50\% relative humidity levels (see Figure 15). It was observed that $50 \mathrm{ppm}$ of toluene in the vapor resulted in a chemicapacitor response of $700 \mathrm{aF}$, well above the sensor resolution. This was the first time such a high performance had been reported for chemicapacitors on flexible plastic substrates under normal humidity conditions. This low-power, low-cost, sensor system could evolve to be well suited for stand-alone applications like RFID tags where separate sensing polymers can be used to detect individual VOCs. However, the widespread use of silicon substrates in conventional microelectronics fabrication may act as an obstacle to widespread uptake of these new technologies until they show clear performance or cost improvements. Advances in the area of low-cost, low-temperature printed electronics may help to alleviate such barriers. 


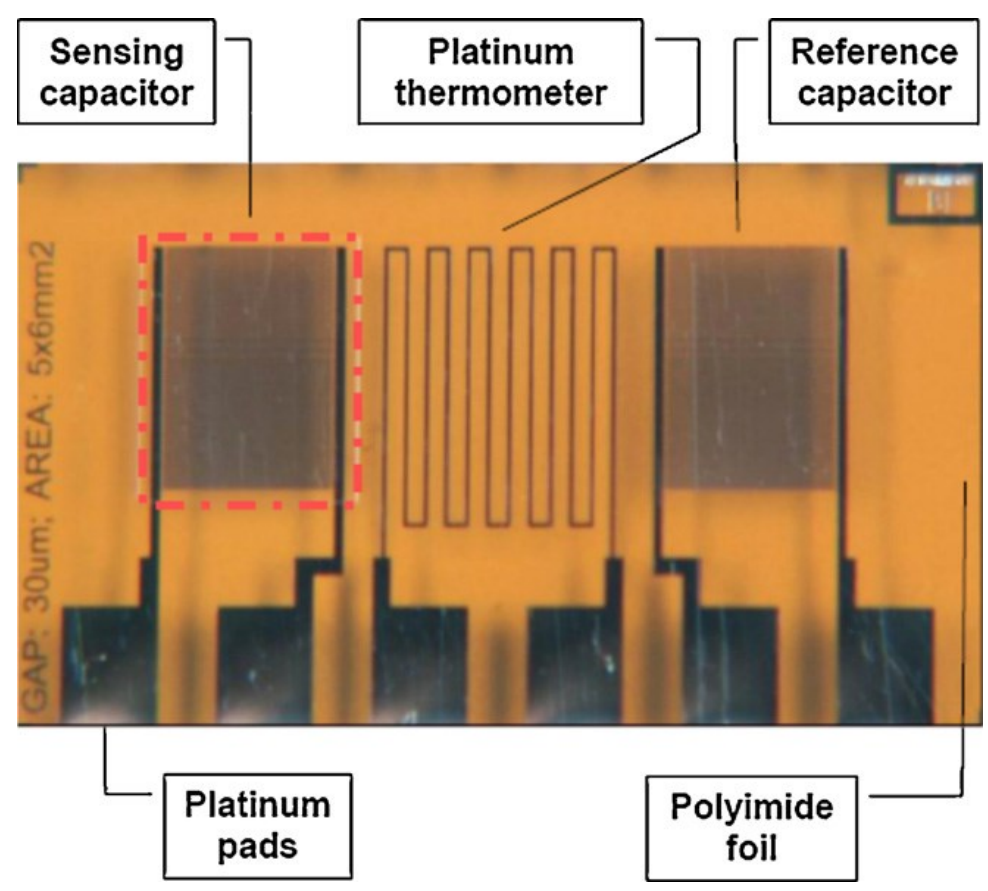

(a)

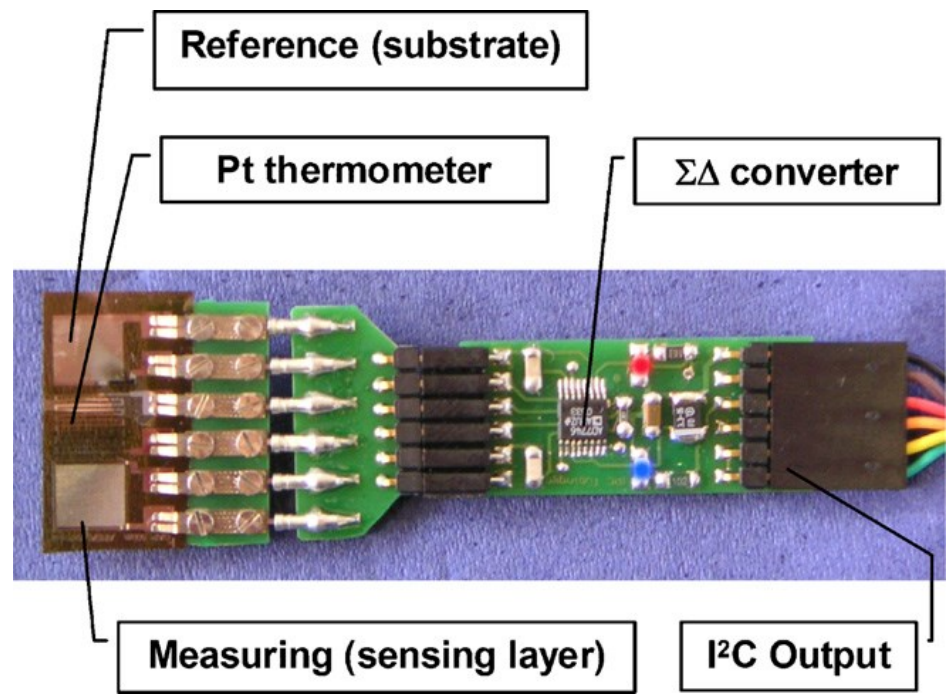

(b)

Figure 14. (a) Chemicapacitor sensing system formed on flexible polyimide substrate. (b) Chemicapacitor measurement and reference sensors integrated with a CDC and computer interfacing technology. Reproduced with permission from Oprea et al. [80]. Copyright Elsevier 2009. 


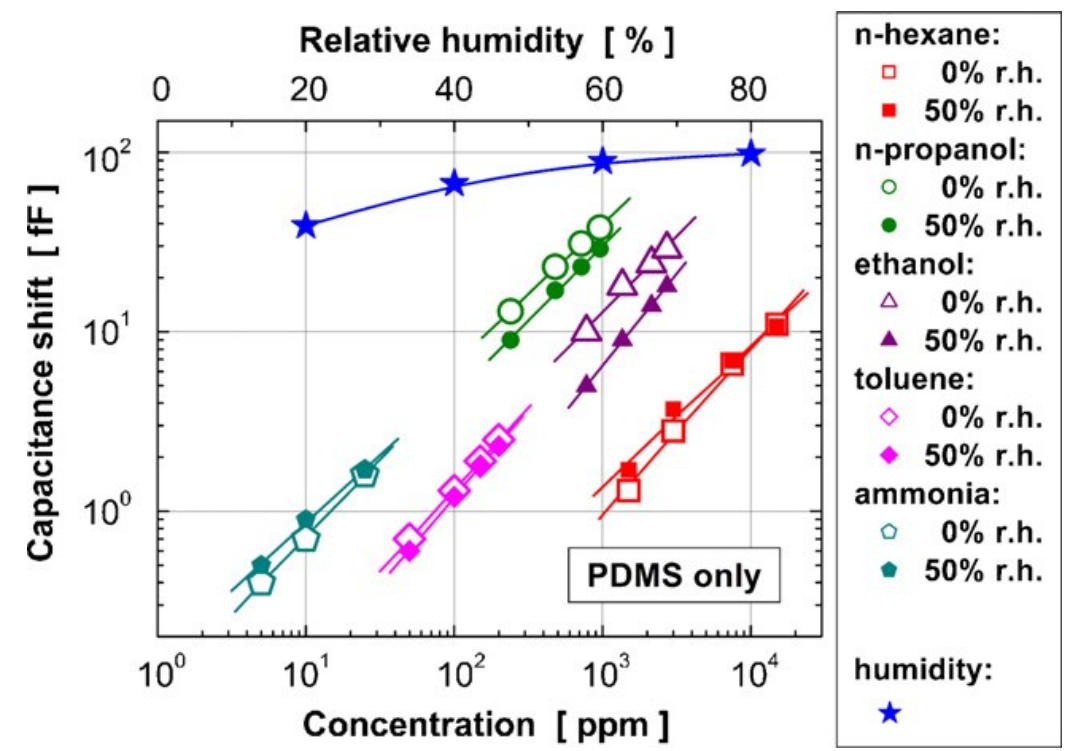

Figure 15. Detection of a range of VOCs in 0 and $50 \%$ relative humidity using chemicapacitor sensors on flexible substrates. Reproduced with permission from Oprea et al. [80]. Copyright Elsevier 2009.

\section{Review of Parallel Plate Chemicapacitors}

\subsection{Parallel Plate Chemicapacitor Gas Sensors}

The majority of chemicapacitor sensors reported to date have used the planar IDE platform due to its ease of manufacture and coating, but parallel plate chemicapacitor sensors have been also been developed. In the early $1990 \mathrm{~s}$, as an alternative to commercially developed optical $\mathrm{CO}_{2}$ sensors and concentration cells incorporating solid electrolytes, Ishihara et al [81] investigated the development of a parallel plate chemicapacitor $\mathrm{CO}_{2}$ sensor using mixed oxides as a step towards simplification, miniaturization, and cost reduction. Oxides have advantages of long term stability and can be used to measure $\mathrm{CO}_{2}$ through a dielectric change of the oxide. However, fabrication of the mixed oxide disks required extended high temperature processing. A combination of $\mathrm{BaTiO}_{3}$ and $\mathrm{CuO}$ in the form of a $13 \mathrm{~mm}$ diameter disk had electrodes formed on opposite sides of the disk using Ag paste, together with embedded Pt lead wires. With this system $\mathrm{CO}_{2}$ concentrations up to $2 \%$ in dry air could be detected in a linear fashion at a frequency of $50 \mathrm{kHz}$ for maximum sensitivity. For reaction rate between a $\mathrm{CO}_{2}$ gas and a solid oxide increases with temperature and for $\mathrm{CuO}-\mathrm{BaTiO}_{3}$ reached a maximum sensitivity at $729 \mathrm{~K}$, with response time of 30 seconds. For a higher $\mathrm{CO}_{2}$ concentration range $\mathrm{NiO}-\mathrm{BaTiO}_{3}$ showed a linear response between 2 and $20 \% \mathrm{CO}_{2}$.

Use has also been made of molecular sieves $\left(\mathrm{AlPO}_{4}-5\right)$ as a thin film (circa $200 \mathrm{~nm}$ thickness) dielectric in early parallel plate chemicapacitors [82]. This system was demonstrated to sense small gaseous molecules that could selectively pass through the 7 Angstrom pores, such as $\mathrm{CO}_{2}$ to a lower concentration of $50 \mathrm{ppm}$ and $\mathrm{CO}$ to $100 \mathrm{ppm}$, which compared well with commercial CO sensors of the time. The synthesis of $\mathrm{AlPO}_{4}-5$ films required bulk material processing over many hours followed by laser ablation of the film onto the titanium nitride electrodes at temperatures up to $350^{\circ} \mathrm{C}$. Water 
vapor was found to give a comparatively very small change in signal, and since the sensing film is stable up to $700^{\circ} \mathrm{C}$ a properly designed sensor could find applications in the detection of combustible exhaust gases.

\subsection{Parallel Plate Chemicapacitor VOC Sensors}

Parallel plate chemicapacitors have been developed on flexible substrates to target VOCs using off-the-shelf polymers and a custom non-polymer (tin oxide) sensing film. Zampetti et al. [83] used a flexible polyimide foil (see Figure 16) as the base of a parallel plate capacitor fabricated by low temperature poly-silicon process as a route to lower cost sensors. Three different polymers (PTFE, PMMA, and BCB) were individually sandwiched between a porous upper metal plate and a lower metal plate upon a thin $(8 \mu \mathrm{m})$ polyimide base (see Figure 17). In this sensor the conversion of capacitance changes to an output signal was achieved with an integrated ring oscillator circuit also fabricated on the polyimide base. The ring oscillator consisted of five inverter gate stages and oscillates with a period $\tau_{\text {out }}$ given by

$$
\tau_{\text {out }}=(N-1) \tau_{\text {inv }}+\tau_{\text {sens }}
$$

where $\mathrm{N}$ and $\tau_{\text {inv }}$ are the number and propagation delay of each gate. $\tau_{\text {sens }}$ is the delay of the last gate which is also attached to the sensing capacitor. Thus, changes in the sensing capacitance (induced by a changing VOC atmosphere) will affect the time constant and the delay of this gate and result in a shift in oscillation frequency (equal to the inverse of $\tau_{\text {out }}$ ). Under dry nitrogen carrier gas, the quoted limit of detection was $620 \mathrm{ppm}$ for ethanol vapor and $240 \mathrm{ppm}$ for methanol vapor. PCA was also used to demonstrate the ability to discriminate between methanol and ethanol vapors. No frequency shifts were induced with forced substrate bending indicating the sensor reliability on this flexible substrate. The polymers used, however, are known to have a sensitivity to humidity changes and to overcome this the authors suggest methods to exclude humidity such as a humidity trap. 


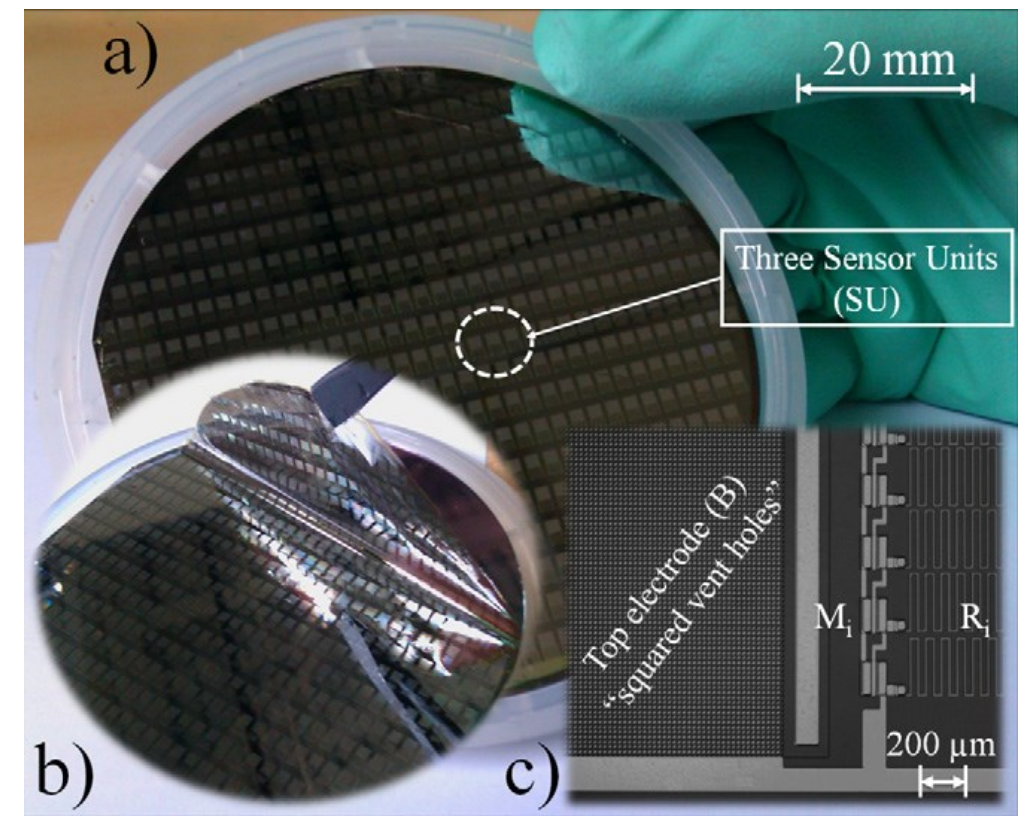

Figure 16. (a) Chemicapacitor sensors formed on a flexible polyimide substrate upon a rigid silicon wafer. (b) Removal of thin flexible substrate (containing up to 400 sensors) from wafer. (c) Close up view of individual sensors (area $1.5 \mathrm{~mm}^{2}$ ) showing porous top electrode. Reproduced with permission from Zampetti et al. [83]. Copyright Elsevier 2011.

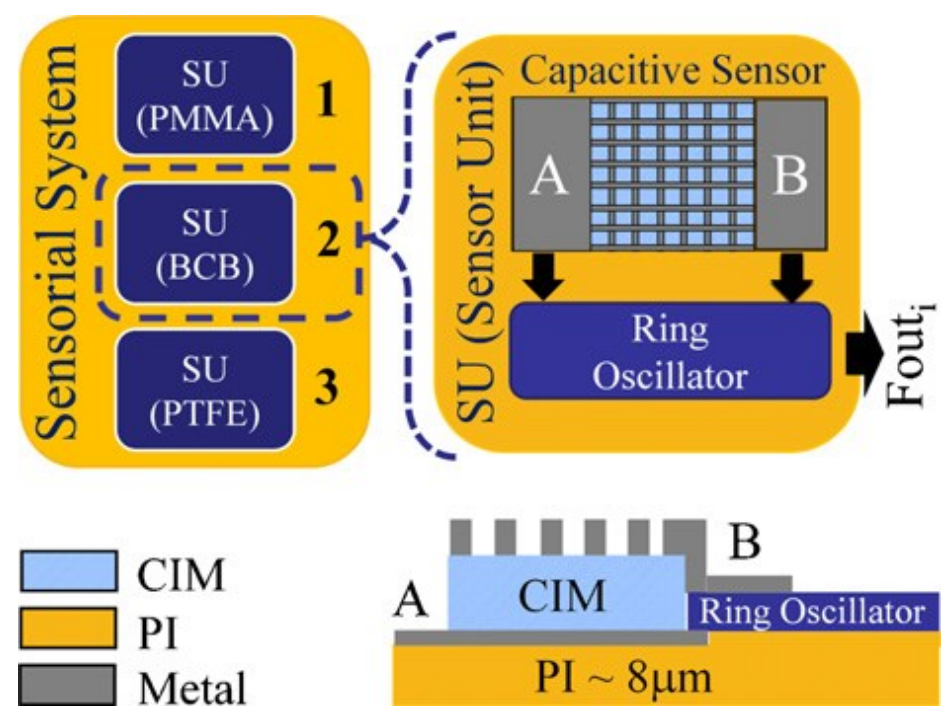

Figure 17. Integrated ring oscillator circuit with a chemicapacitor upon a flexible polyimide substrate. The sensor unit (SU) comprises a parallel plate capacitor with a porous upper plate (B) and a chemically interactive material (CIM). The ring oscillator circuit converts the sensor capacitance into an oscillation frequency. Reproduced with permission from Zampetti et al. [83]. Copyright Elsevier 2011.

A wireless interrogated parallel plate chemicapacitor was also formed on a polyimide substrate using a sensing film composed of tin oxide $\left(\mathrm{SnO}_{2}\right)$ nanoparticles [84]. The bottom plate of the capacitor was attached to a microstrip antenna and the top plate was linked to the antenna ground (see 
Figure 18). An insulating coating of cellulose acetate on the bottom plate prevented short-circuiting. Increasing the concentration of ethylene vapor from 0 to $100 \mathrm{ppm}$ around the sensor resulted in a decrease in the measured capacitance due to dielectric changes in the sensing film.

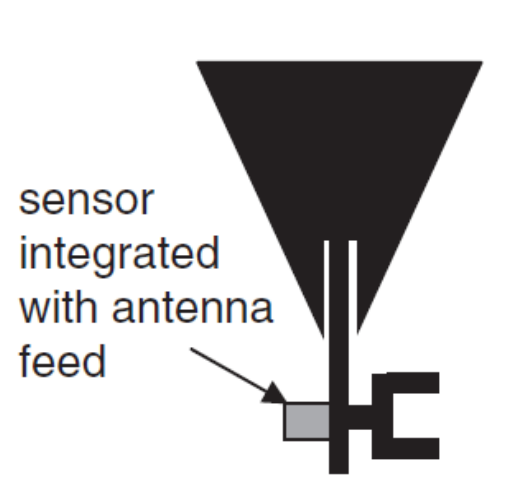

(a)

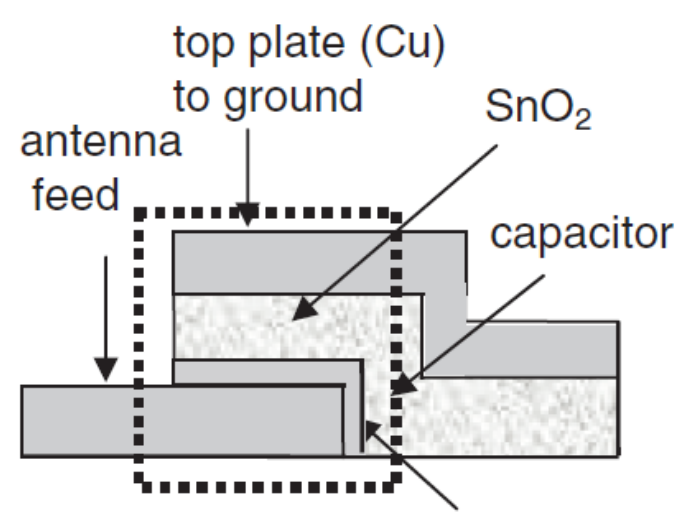

cellulose acetate

(b)

Figure 18. (a) Microstrip antenna of wirelessly addressed sensor attached directly to bottom plate of a parallel plate capacitor. (b) Topology of chemicapacitor with $\mathrm{SnO}_{2}$ sensing layer for the detection of ethylene vapors [84]. Reproduced by permission of The Institution of Engineering and Technology.

A network analyzer with a receiving antenna located at a distance of $16 \mathrm{~cm}$ from the sensor was then used to wirelessly detect the resonance frequency of the sensor. It was observed that decreasing the sensor capacitance was accompanied by a shift to a higher resonance frequency (see Figure 19) and also a reduction in the return loss at this frequency from the sensor. The authors noted that by modifying the design parameters of the sensor the resonance frequency of $961 \mathrm{MHz}$ can be tailored to $915 \mathrm{MHz}$ to match UHF Gen2 RFID systems.

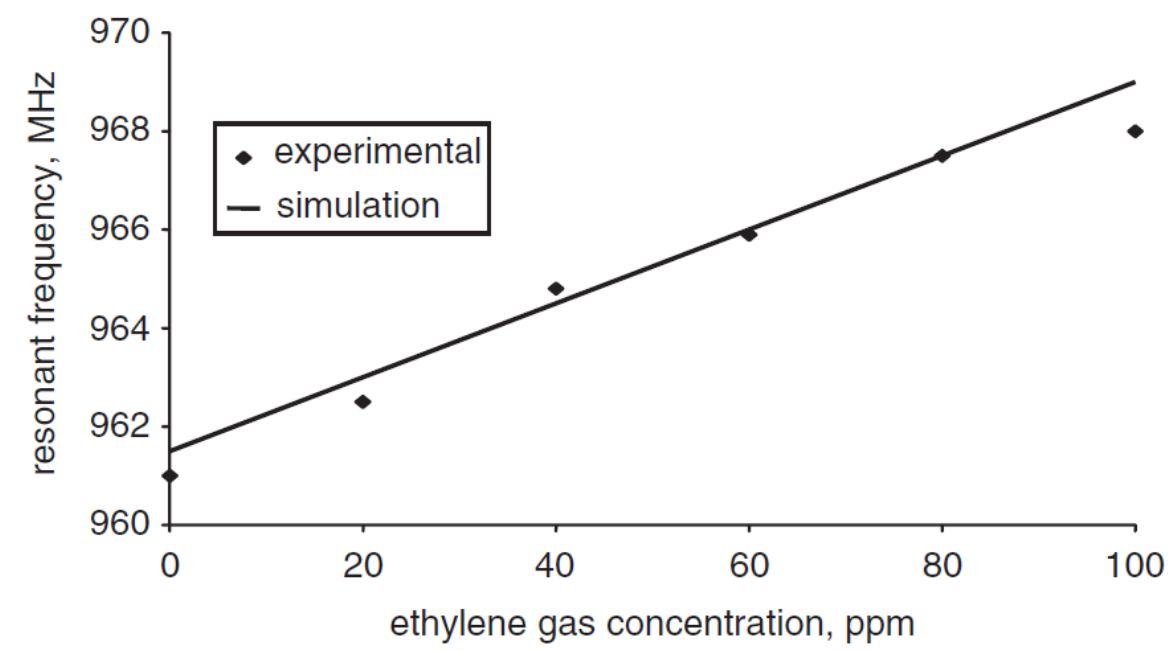

Figure 19. Measured change in resonance frequency due to increasing ethylene concentration with corresponding simulation data [84]. Reproduced by permission of The Institution of Engineering and Technology. 
Finally, a non-standard parallel plate chemicapacitor sensor format with the sensing polymer outside the capacitor plates was also investigated by Chatzandroulis et al. [85]. By fabricating a thin (less than $4 \mu \mathrm{m}$ ) silicon membrane coated on its upper side with a polymer dielectric ( $\mathrm{Si} /$ polymer bimorph) that swells in the presence of target molecules, the authors created a parallel plate chemicapacitor with one moving plate as the sensing mechanism. This flexible membrane was situated $1 \mu \mathrm{m}$ above a fixed silicon substrate and upon deflection the distance between the silicon electrodes changed and thus induced a change in capacitance of the parallel plate structure (see Figure 20). A range of polymers were evaluated for their transduced capacitance changes to vapors of methanol, ethanol, and water individually introduced to a test chamber via a nitrogen carrier gas. In all cases water vapor produced a significantly larger response than either methanol or ethanol, whilst the response to methanol was higher than that of ethanol due the smaller methanol molecules' ability to more easily diffuse into the polymers. For example, in the case of a poly-hydroxy-ethyl-methacrylate coated sensor, a methanol concentration of $34,400 \mathrm{ppm}$ produced a capacitance change of over $1.1 \mathrm{pF}$ whilst for $19,400 \mathrm{ppm}$ of ethanol only a $0.3 \mathrm{pF}$ change occurred, both with a relatively slow response time of around 30 minutes for this device structure.

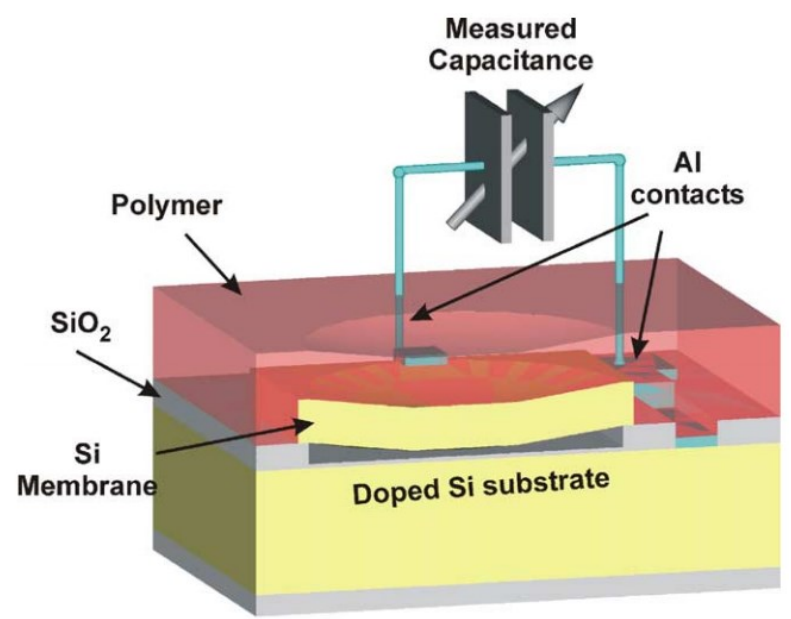

Figure 20. Sensing polymer coated on a flexible silicon diaphragm situated above a fixed silicon electrode forming a parallel plate chemicapacitor sensor for VOCs. Reproduced with permission from Chatzandroulis et al. [85]. Copyright Elsevier 2004.

\section{Moving Chemicapacitor VOC Sensors Beyond the Laboratory}

In comparison to chemicapacitor humidity sensors, the commercial exploitation of polymerbased chemicapacitor VOC sensors has proven very much slower due to the cross-sensitivity of polymers to environmental interferents and the steps needed to overcome the cross-sensitivity. Nevertheless, this process has begun. Seacoast Science Inc. (California, USA) since 2003 has developed commercial chemicapacitor technology for the detection of a range of VOCs, industrial chemicals, and warfare agents [86]. These parallel plate chemicapacitors (see Figure 21(a)) on silicon wafers are fabricated in polycrystalline silicon (situated on an insulating silicon nitride layer) using micro-machining technology (MUMPS) that allows the device formation, followed by subsequent 
inkjet deposition of the sensing polymer between the plates. This methodology lends itself to the formation of arrays of relatively smaller devices with good reproducibility between devices (since the parallel plate format has a defined polymer volume). Ten sensors (with either a 0.75 or $2 \mu \mathrm{m}$ gap between the plates) can be formed on a single $5 \mathrm{~mm} \times 2 \mathrm{~mm}$ chip (see Figure 21(b)). Anchoring posts are attached to the top plate to reduce plate deformation due to polymer swelling. The top plate had a pattern of $2 \mu \mathrm{m}$ diameter holes etched through it to allow analyte molecules to reach the polymer. The fabricated sensors have been interfaced to a custom charge/discharge readout circuit in a single dualin-line package [69] and integrated with a 12-bit analog to digital converter and a microcontroller.

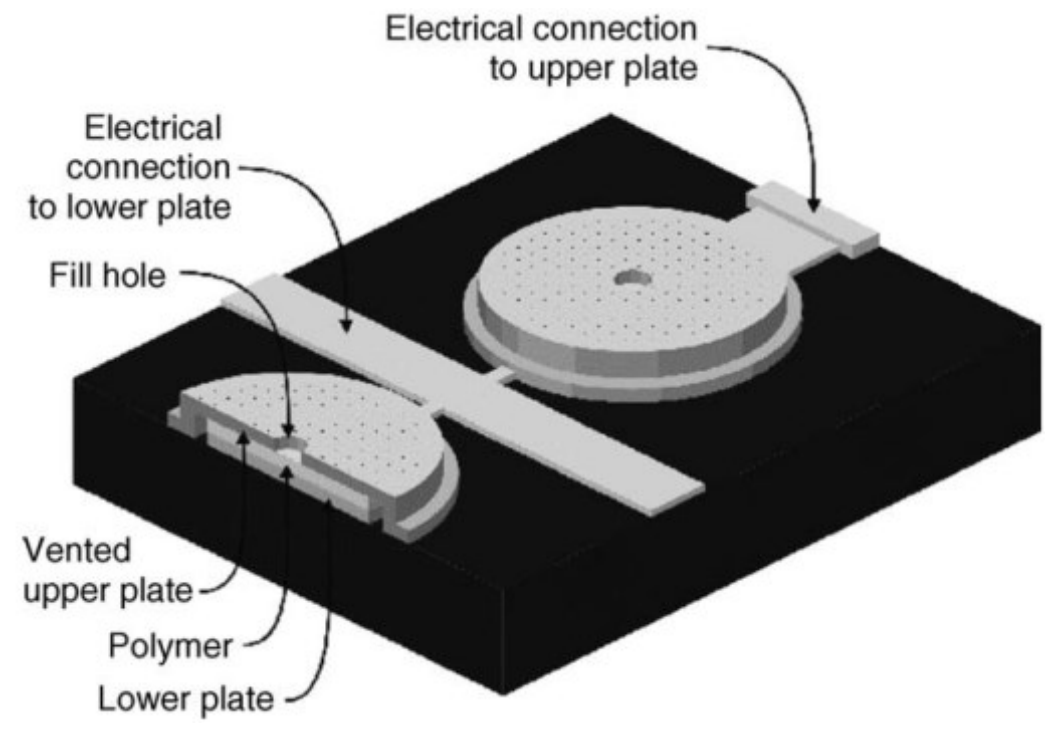

(a)

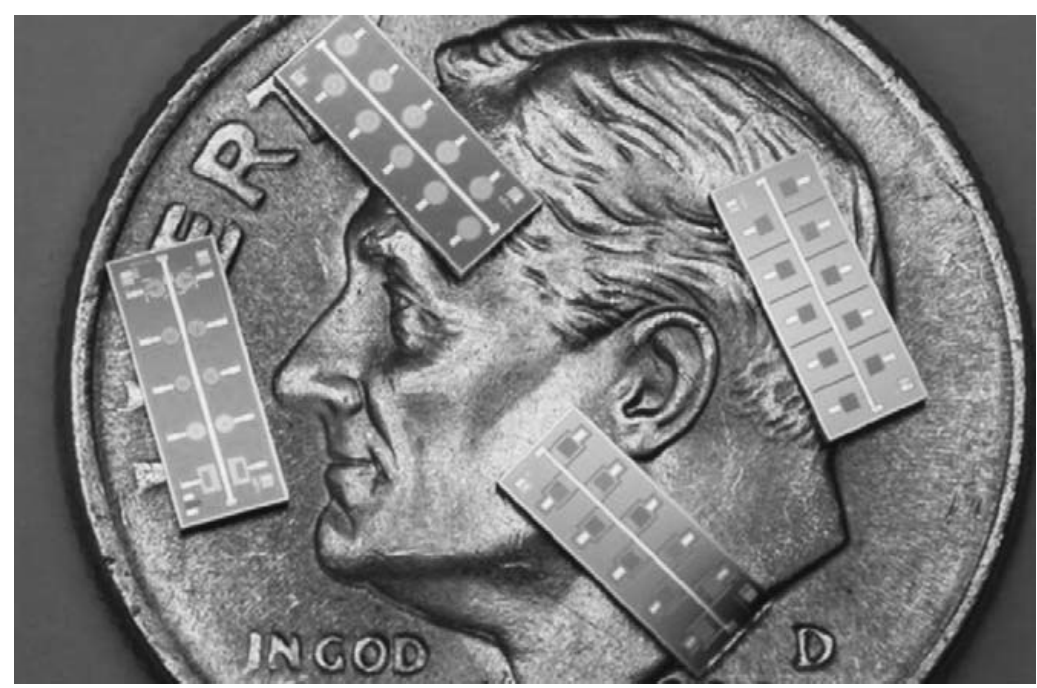

(b)

Figure 21. (a) Structure of polymer-filled parallel plate chemicapacitors developed by Seacoast Science Inc. (b) Ten individual chemicapacitors upon a single chip demonstrating the extent of miniaturization achievable. Reproduced with permission from Mlsna et al. [87]. Copyright Elsevier 2006. 
A range of off-the-shelf polymers have been investigated by Seacoast Science Inc. for their selectivity in forming weak reversible bonds with target molecules. These developed sensors demonstrated $\mathrm{ppb}$ detection levels of chemical warfare agents and explosive by-products such as DMMP and nitrotoluene, with a sensor response to $800 \mathrm{ppb}$ DMMP over twice the response to $40 \%$ water vapor [86]. It was also observed that the performance of the chemicapacitor was dependent on temperature changes due to the changing volatility of target vapors. In practical applications of the technology the microcontroller could also combine signals from the chemicapacitor with readings from independent humidity and temperature sensors to calibrate out these influences. Other industrial VOCs (such as benzene and toluene) could also be detected at below 100 ppm levels. Depending on the rigidity of the polymers and the size of the target analyte the $90 \%$ response time (in $0 \%$ humidity) ranged from 20 secs to several minutes, with the reverse response to the removal of the target usually being a longer process. This sensor technology was further developed into a small, low-power system (see Figure 22) [87].

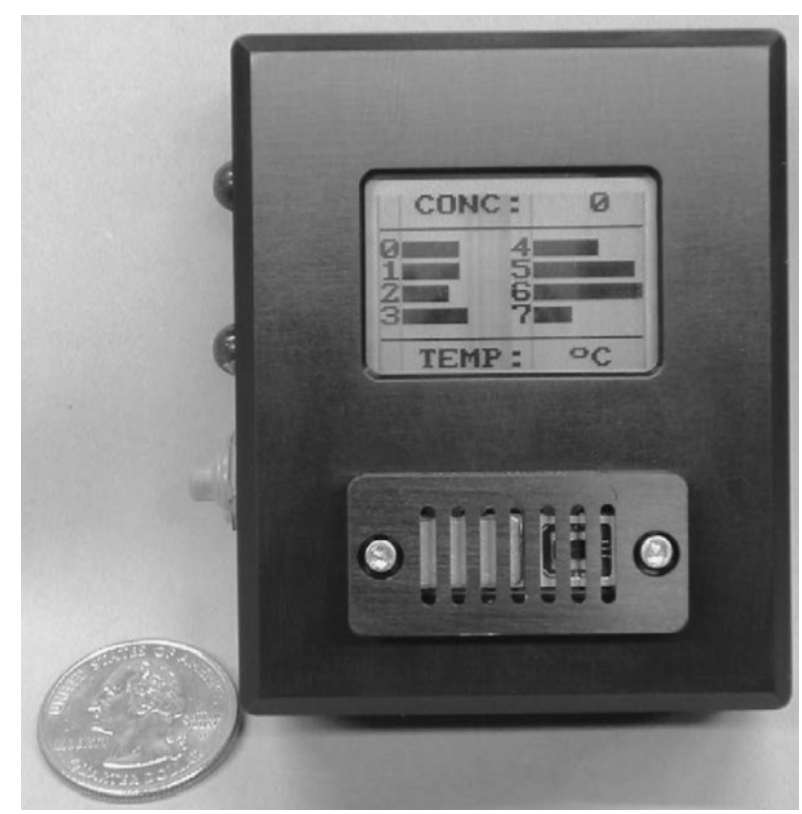

Figure 22. Compact, battery-operated, chemical warfare agent detection system developed by Seacoast Science Inc. Reproduced with permission from Mlsna et al. [87]. Copyright Elsevier 2006.

Over the last decade this technology has progressed to demonstrate the detection of a range of molecules in a number of different applications. The LOD of several toxic industrial chemicals and chemical warfare agents, such as hydrogen cyanide (a blood agent) to $25 \mathrm{ppm}, 2,6$-DNT (an explosive) to $0.002 \mathrm{ppm}$, carbaryl (a pesticide) to $0.0006 \mathrm{ppm}$, styrene oxide (a toxic chemical) to $0.44 \mathrm{ppm}$, and tabun (a nerve agent) to $0.05 \mathrm{ppm}$ has been achieved [87]. Methyl salicylate (a chemical used to test the effectiveness of protective garments) was detected to sub-ppm levels [88]. Additionally, a sensor system was developed to measure small concentrations of ethanol (0.1 to $2 \%)$ in water by using a hydrophobic membrane filter that allowed ethanol vapor from the liquid to pass through onto the sensor [89]. The ability to monitor within aqueous environments opens up new areas for the deployment of chemicapacitors. 
Response times for these parallel plate chemicapacitors have also been improved by increasing the size and number of openings in the upper plate to allow faster vapor diffusion, but are still much slower than IDE chemicapacitors (see Figure 9) fabricated by Seacoast Science [87]. However, the parallel plate chemicapacitors give a higher sensitivity. Table 1 summarizes the LOD of some of the parallel plate chemicapacitors developed by Seacoast Science targeting vapors of explosives and taggants for explosives.

Table 1 Theoretical LOD's of parallel plate chemicapacitors developed by Seacoast Science Inc. Reproduced with permission from Patel et al. [69]. Copyright 2005 Society of Photo Optical Instrumentation Engineers.

\begin{tabular}{|c|c|c|c|}
\hline Analyte & LOD (ppm) & Polymer/Sensor & $\begin{array}{l}\text { Temperature } \\
\left({ }^{\circ} \mathbf{C}\right)\end{array}$ \\
\hline \multirow[t]{2}{*}{ DMDNB } & 0.02 & OV225 & 25 \\
\hline & 0.08 & PECH & 25 \\
\hline \multirow[t]{2}{*}{ o-Nitrotoluene } & 0.5 & OV225 & 20 \\
\hline & 0.4 & PECH & 20 \\
\hline \multirow[t]{2}{*}{ 2,6-DNT } & 0.001 & OV225 & 20 \\
\hline & 0.002 & $\mathrm{PECH}$ & 20 \\
\hline \multirow[t]{2}{*}{ 2,4-DNT } & 0.01 & OV225 & 20 \\
\hline & 0.03 & PECH & 20 \\
\hline 1-Nitropropane & 10 & OV225 & 25 \\
\hline \multirow[t]{2}{*}{ Nitrobenzene } & 0.4 & OV225 & 20 \\
\hline & 0.1 & OV225 & 10 \\
\hline
\end{tabular}

Subsequently, Seacoast Science developed an automated sensor for on-site contamination. The device incorporated a coated pre-concentrator tube that selectively absorbs target molecules from the vapor stream which are then released by heating the tube. The pre-concentrator improved the detection limit by up to 2000 times. The sensor consisted of an array of four pairs of chemicapacitors coated with four different polymers [90]. An additional pair of sensors was coated with a composite of polyvinyl acetate and polyethylenimine polymers to form humidity sensors. This was demonstrated in a standalone subsurface automated system for continuous monitoring of soil and groundwater contamination. Such measurements are traditionally achieved by periodic sampling and off-site laboratory analysis. Initial targets were chlorinated hydrocarbons and benzene, with detection to around $1 \mathrm{ppm}$ achieved in just 8 minutes. For longer collection times the detection of ppb levels of benzene and trichloroethane was demonstrated. A system was later shown to achieve standalone operation for over 500 sampling and analysis sequences [91].

The company has gone on to commercialize low-cost gas chromatography (GC) kits [92] for academia and a field-portable GC system with applications in agriculture, quality control, security, manufacturing process monitoring and water quality [93]. These systems have automatic sampling and operate in room air and thus require no carrier gases. 


\section{Conclusions}

The desire and regulatory enforcement of the detection of a wide range of gases and vapors in the environment has compelled researchers to seek technologies that will evolve detection systems from costly and bulk equipment to smaller cost-effective systems that can be more widely deployed and, ideally, networked. Such a technology would increase the amount and speed of information available on the levels of environmental molecules, which has applications from manufacturing processes to monitoring the release of pollutants, all of which can improve the quality of life. The need to measure a diverse range of target species can potentially be realized by developing capacitance measurements on thin non-conducting polymer sensing films (chemicapacitors) that can form a technology that is low-cost, miniature and readily combined with integrated electronic circuits.

Unlike sensors which measure a change in the conductivity of a conductive polymer (chemiresistor) capacitance uses a non-dissipative measurement technique facilitating lower power sensor systems (even zero power autonomous sensors). Also, as sensors are miniaturized the sensitivity of resistive measurements usually decreases [94]. In parallel the electronics community has developed single IC chips known as capacitance to digital converters (CDCs) which permit determination of capacitances with a resolution of a few attoFarads at $\mathrm{kHz}$ measurement rates. This has allowed capacitive sensing technologies to spread throughout society from vehicle sensors to touchscreen displays.

However, in the field of chemicapacitors this success has been slow to be repeated for the measurement of VOCs and gases due to inherent issues with this technology, specifically non-specific absorption processes. These sensors commonly employ a polymer sensing film which has a low dielectric constant and results in a large cross-sensitivity to humidity, as well as sensitivity to interferent VOCs other than the sensor target. In addition temperature changes (which affect the VOC volatility) affect the stability and sensitivity of the chemicapacitor sensor. As such, chemicapacitor sensors have been successfully (commercially) developed for humidity sensing for decades, but chemicapacitors for VOCs in general are yet to follow at the same pace. Over the years a lot of research has gone into overcoming these environmental impact issues using a range of measurement formats.

In the 1990's chemicapacitors were developed for several gas targets using non-polymer sensing films and achieved sensitivity to ppm levels and response times of tens of seconds, and researchers also began to address issues of interference from humidity and temperature changes by integrating resistive heaters on the sensor platform to reduce temperature fluctuations across the sensing area to a few percent. However, the power dissipation from the heater alone was a few hundreds of $\mathrm{mW}$. Attention subsequently turned to polymers materials to create lower powered sensors for VOCs. Researchers demonstrated the detection of target VOCs to ppm concentrations and also began to interface these devices with integrated circuits using established CMOS fabrication techniques. It was found that by the selective choice of polymer sensing film that the sensor response to water vapor could be made less than to the target VOCs. However, often during evaluation of these sensors the devices were tested within humidity controlled environments. By early 2000s this technology had progressed to a demonstration of a battery-operated handheld device with LCD display, but as always, water vapor and other interferents would cause unwanted signal changes. 
In parallel studies, polymer material absorption processes and the geometry of IDE revealed a sensor dependent behavior that indicated methods that could differentiate target molecules. Also, by identifying different polymer affinities for different vapors arrays of chemicapacitors were developed with different polymer sensing films and numerical algorithms such as Principal Component Analysis could be employed for individual target vapor recognition within a mixture of VOCs. In addition, the differential response to water, sensor temperature modulation and frequency spectroscopy were evaluated as methods to reduce humidity interference.

Progress in these areas resulted in an array of chemicapacitor sensors integrated with a commercial CDC which could discriminate individual analytes and binary mixtures. With the selection of a hydrophobic polymer the detection of low concentrations (to tens of ppm) of target VOCs was possible in the presence of normal levels of humidity (up to $50 \% \mathrm{RH}$ ). These developed chemicapacitor sensors were successfully applied to grain storage and fermentation process applications.

Alternative lower cost methods for coating sensors using electrochemical deposition are also being investigated. This technology polymerizes monomers from a solution onto a localized area of a substrate. This can allow the single step coating of numerous devices on a silicon wafer in a matter of minutes. In addition, by chemically engineering the monomers, polymers can be formed with a much higher affinity to sensor targets than off-the-shelf polymers.

In the area of security small stand-alone sensors are greatly desired as an alternative to sniffer dogs and bulk expensive technology for the detection of explosives. Naturally, much work has been done in this area. Polymer-based parallel plate chemicapacitors for explosive and warfare agents have been successfully developed with excellent sensitivity (parts per billion). Combined with reference sensors for humidity and temperature, an integrated microcontroller, and numerical software these have been created as battery powered stand-alone modules and are now commercial products.

Compared to traditional solid substrates, flexible polymer substrates (such as polyimide) can be lighter in weight, foldable, and reduce manufacturing costs of devices facilitating production on a larger scale compared to traditional solid substrates. Researchers developing chemicapacitors have demonstrated techniques to alleviate humidity and temperature instability inherent in these polymer substrates and the integration with CDCs to achieve high performance sensing of VOCs under normal atmospheric conditions. If the sensor signals were acquired at 1 minute intervals, this reduced the dissipated power for each capacitor to picoWatts whilst attaining attoFarad resolution.

The non-dissipative nature of capacitive sensing has also encouraged research into the development of autonomous low power $(<100 \mu \mathrm{W})$ sensors and wireless interrogation techniques. Researchers have demonstrated devices with non-polymer sensing films $\left(\mathrm{CNTs}\right.$ and $\left.\mathrm{SnO}_{2}\right)$ for the measurement of gas and VOC ethylene (with the sensor formed on a polyimide substrate). Nevertheless, to date, commercial RFID-type tags employing polymers are still limited to measurements of humidity and temperature (for example, the Hygro-Fenix-H221 (Farsens S. L.)). More recently, however, a polymer-based chemicapacitor sensor combined with a RF resonant sensor was shown to be able to measure ethanol using an RF wave with time and amplitude modulation.

Looking to the future, the non-specific absorption processes inherent in polymers, which has hampered the development and deployment of chemicapacitor sensor systems, may actually hold the 
best opportunity for this platform. Sensor arrays can mimic the olfactory system found in nature where numerous receptors are not selective for specific molecules, but are stimulated by a range of vapors and post-processing of these signals using pattern recognition is utilized [95]. The low-cost of forming arrays of polymer-based sensors (especially on plastic substrates) combined with on-going reductions in power consumption, miniaturization, and increasing capabilities of algorithmic processing of electronic circuits will be an enabler for this format. Having a numerous array of sensors, each with a varying selectivity to a wide range of target vapors, would negate the need for overly engineering a sensor specifically for one target (to the exclusion of others) and would actually expand the range of targets and capabilities in one sensor system.

\section{References}

1. Kumar P, Morawska L, Martani C, Biskos G, Neophytou M, Di Sabatino S, Bell M, Norford L and Britter R 2015 The rise of low cost sensing for managing air pollution in cities Environment International 75 199-205.

2. World Health Organisation/Europe. Available online: http://www.euro.who.int/_data/assets/pdf_file/0006/189051/ Health-effects-of-particulate-matterfinal-Eng.pdf.

3. City of London Corporation. Available online: https://www.cityoflondon.gov.uk/business/environmental-health/environmental-protection/airquality/Documents/Impacts-Of-Air-Pollution-On-Health.pdf.

4. Joshi, S M 2008 The sick building syndrome Indian J Occup Environ Med. 12 61-64.

5. Centers for Disease Control and Prevention (USA). Available online: http://www.cdc.gov/nceh/clusters/Fallon/.

6. Tunsaringkarn T, Prueksasit T, Kitwattanavong M, Siriwong W, Sematong S, Zapuang K and Rungsiyothin A 2012 Cancer risk analysis of benzene, formaldehyde and acetaldehyde on gasoline station workers J. Environ. Eng. Ecol. Sci. 1 1-6.

7. National Cancer Institute (USA). Available online: http://www.cancer.gov/aboutcancer/causes-prevention/risk/substances/formaldehyde/formaldehyde-fact-sheet (accessed on 12 December 2015).

8. The Collaborative on Health and the Environment (USA). Available online: http://www.healthandenvironment.org/docs/CHE_Toxicants_and_Disease_Database.pdf.

9. Wilson A D 2012 Review of electronic-nose technologies and algorithms to detect hazardous chemicals in the environment Procedia Techn. 1 453-463.

10. United States Department of Labor (USA). Available online: http://www.osha.gov.

11. Li S, Simonian A and Chin B A 2010 Sensors for Agriculture and the Food Industry The Electrochemical Society Interface Winter 2010 41-46.

12. Weerakoon K A, Shu J H and Chin B A 2011 A chemiresistor sensor with a poly(3hexylthiophene) active layer for the detection of insect infestation at early stages IEEE Sensors $J .11$ 1617-1622. 
13. Jonsson M and Anderson P 2007 Emission of oilseed rape volatiles after pollen beetle infestation behavioral and electrophysiological responses in the parasitoid pharadis morionellus Chemoecology 17 201-207.

14. Deisingh A K, Stone D C and Thompson M 2004 Applications of electronic noses and tongues in food analysis Int. J. Food Sci. Tech. 39 587-604.

15. Queralto N, Berliner A N, Goldsmith B, Martino R, Rhodes P and Lim S H 2014 Detecting cancer by breath volatile organic compound analysis: A review of array-based sensors $J$ Breath Res. 8 027112.

16. Singh S 2015 Sensors - an effective approach for the detection of explosives J. Hazard. Mater. 144 15-28.

17. Hanson R K, Spearrin R M and Goldenstein C S Spectroscopy and optical diagnostics for gases. Springer International Publishing, Cham, Switzerland, 2015.

18. Creaser C S, Griffiths J R, Bramwell C J, Noreen S, Hillb C A and Thomas P 2004 Ion mobility spectrometry: A Review. Part 1 . Structural analysis by mobility measurement Analyst 129 984-994.

19. Westhoff M, Litterst P, Freitag L, Urfer W, Bader S and Baumbach J I 2009 Ion mobility spectrometry for the detection of volatile organic compounds in exhaled breath of patients with lung cancer: Results of a Pilot Study Thorax 64 744-748.

20. Smiths Detection (2011) SABRETM 5000 Handheld trace detector for explosives, chemical agents and toxic industrial chemicals or narcotics. Available Online: http://www.militarysystemstech.com/files/militarysystems/supplier_docs/Sabre5000.pdf (accessed on 19 July 2015).

21. Hök B, Blückert A and Löfving J 2000 Acoustic gas sensor with ppm resolution Sensor Review 20 139-142.

22. Riegel J and Härdtl K H 1990 Analysis of combustible gases in air with calorimetric gas sensors based on semiconducting BaTiO3 ceramics Sens. Actuators B Chem. 1 54-57.

23. Fine G F, Cavanagh L M, Afonja A and Binions R 2010 Metal oxide semi-conductor gas sensors in environmental monitoring Sensors 10 5469-5502.

24. Dong Y, Gao W, Zhou Q, Zheng Y and You Z 2010 Characterization of the gas sensors based on polymer-coated resonant microcantilevers for the detection of volatile organic compounds Anal. Chim. Acta 671 85-91.

25. Si P, Mortensen J, Komolov A, Denborg J and Møller P J 2007 Polymer coated quartz crystal microbalance sensors for detection of volatile organic compounds in gas mixtures Anal. Chim. Acta $\mathbf{5 9 7} 223-230$.

26. Sayagoa I, Fernándeza M J, Fontechaa J L, Horrilloa M C, Verab C, Obietab I and Bustero I 2012 New sensitive layers for surface acoustic wave gas sensors based on polymer and carbon nanotube composites Sens. Actuators B Chem. 175 67-72.

27. Olthuis W, Sprenkels A J, Bomer J G, and Bergveld P 1997 Planar interdigitated electrolyteconductivity sensors on an insulating substrate covered with $\mathrm{Ta}_{2} \mathrm{O}_{5}$ Sens. Actuators B Chem. 43211 216. 
28. Pavinatto F J, Paschoal C W A and Arias A C 2015 Printed and flexible biosensor for antioxidants using interdigitated ink-jetted electrodes and gravure-deposited active layer Biosens. Bioelectron. 67 553-559.

29. Singh K V, Bhura D K, Nandamuri G, Whited A M, Evans D, King J and Solanki R 2011 Nanoparticle-enhanced sensitivity of a nanogap-interdigitated electrode array impedimetric biosensor Langmuir 27 13931-13939.

30. Schaur S and Jakoby B 2011 A numerically efficient method of modeling interdigitated electrodes for capacitive film sensing Procedia Engineering 25 431-434.

31. Igreja R and Dias C 2006 Dielectric response of interdigital chemocapacitors: The role of the sensitive layer thickness Sens. Actuators B Chem. 115 69-78.

32. Seacoast Science Inc. Available online: http://www.seacoastscience.com/Downloads/Seacoast\%20Science\%20ISOEN\%20Poster.pdf.

33. Igreja R and Dias C J 2004 Analytical evaluation of the interdigital electrodes capacitance for a multi-layered structure Sens. Actuators A Phys. 112 291-301.

34. Boylestad R L 1994 Introductory circuit analysis $7^{\text {th }}$ ed. (New York, USA: Maxwell Publishing Company).

35. Balakrisnan B, Patil S and Smela E 2009 Patterning PDMS using a combination of wet and dry etching J. Micromech. Microengin. 19047002.

36. Cho N B, Lim T H, Jeon Y M and Gong M S 2008 Humidity sensors fabricated with photocurable electrolyte inks using an ink-jet printing technique and their properties Sens. Actuators $B$ Chem. 130 594-598.

37. Vaid T P, Burl M C and Lewis N S 2001 Comparison of the performance of different discriminant algorithms in analyte discrimination tasks using an array of carbon black-polymer composite vapor detectors Anal. Chem. 73 321-331.

38. Aziz P M, Sorensen H V and van der Spiegel 1996 An Overview of Sigma-Delta Converters IEEE Signal Processing Magazine January 61-84.

39. Avramov-Zamurovic S and Lee R D 2009 A high-stability capacitance sensor system and its evaluation IEEE Transactions on Instrumentation and Measurement 58 955-961.

40. Schreier R and Temes G C 2005 Understanding delta sigma data converters (Piscataway, NJ: IEEE Press/Wiley Interscience).

41. Omran H, Arsalan M and Salama K N 2014 An integrated energy-efficient capacitive sensor digital interface circuit Sens. Actuators A Phys. 216 43-51.

42. Otsuka K, Kinoki S, Usui T 1980 Organic polymer humidity sensor Denshi-zairyo 19 68-73.

43. Delapierre G, Grange H, Chambaz B and Destannes L 1983 Polymer-based capacitive humidity sensor: Characteristics and experimental results Sens. Actuators B Chem. 4 97-104.

44. Glenn M C and Schuetz J A June 1985 An IC Compatible polymer humidity sensor Tech. Digest, Transducers '85, Philadelphia, Pa., USA, pp. 217-220.

45. Alberti K, Haas J, Plog C and Fetting F 1991 Zeolite coated interdigital capacitors as a new type of gas sensor Catal. Today 8 509-513.

46. Lin J, Möller S, Obermeier E 1991 Thin-film gas sensors with organically modified silicates for the measurement of $\mathrm{SO}_{2}$ Sens. Actuators B Chem. 5 219-221. 
47. Mutschall D and Obermeier E 1995 A Capacitive $\mathrm{CO}_{2}$ sensor with on-chip heating Sens. Actuators B Chem. 24-25 412-414.

48. Endres H-E, Hartinger R, Schwaiger M, Gmelch G and Roth M 1999 A capacitive $\mathrm{CO}_{2}$ sensor system with suppression of the humidity interference Sens. Actuators B Chem. 57 83-87.

49. Kebabian P L, and Freedman A 2006 Fluoropolymer-based capacitive carbon dioxide sensor Meas. Sci. Technol. 17 703-710.

50. Josse F, Kukas R, Zhou R, Schneider S and Everhart D 1996 AC-impedance-based chemical sensors for organic solvent vapors Sens. Actuators B Chem. 35-36 363-369.

51. Casalini R, Kilitziraki M, Wood D and Petty M C 1999 Sensitivity of the electrical admittance of a polysiloxane film to organic vapors Sens. Actuators B Chem. 56 37-44.

52. Cornila C, Hierlemann A, Lenggenhager R, Malcovati P, Baltes H, Noetzel G, Weimar U and Gijpel W 1995 Capacitive sensors in CMOS technology with polymer coating Sens. Actuators B Chem. 24-25 357-361.

53. Hierlemann A, Lange D, Hagleitner C, Kerness N, Koll A, Brand O and Baltes H 2000 Application-specific sensor systems based on CMOS chemical microsensors Sens. Actuators B Chem. 70 2-11.

54. Steiner F P, Hierlemann A, Cornila C, Noetzel G, Bächtold M, Korvink J G, Gopel W and Baltes H 1995 Polymer coated capacitive microintegrated gas sensor Proc. of Transducers 2 814-817. 55. Hagleitner C, Hierlemann A, Lange D, Kummer A, Kerness N, Brand O and Baltes H 2001 Smart single-chip gas sensor microsystem Nature 414 293-296.

56. Hagleitner C, Lange D, Hierlemann A, Brand O and Baltes H 2002 CMOS single-chip gas detection system comprising capacitive, calorimetric and mass-sensitive microsensors IEEE J. SolidSt Circ. 37 1867-1878.

57. Igreja R and Dias C J 2004 Capacitance response of polysiloxane films with interdigital electrodes to volatile organic compounds Materials Science Forum 455-456 420-424.

58. Igreja R and Dias C J 2006 Organic vapor discrimination using absorption sensitive chemocapacitor arrays Materials Science Forum 514-516 1064-1067.

59. Kitsara M, Goustouridis D, Chatzandroulis S, Chatzichristidi M, Raptis I, Ganetsos Th., Igreja R and Dias C J 2007 Single chip interdigitated electrode capacitive chemical sensor arrays Sens. Actuators B Chem. 127 186-192.

60. Manoli K, Sanopoulou M, Karonis E, Chatzichristidi M, Goustouridis D, Chatzandroulis S and Raptis I 2008 Evaluation of a chemocapacitive sensor array for the detection of vapor analytes and their mixtures Proc. IEEE Sensors 2008 Conference, Lecce, 26-29 Oct 2008, pp. 831-834.

61. Manoli K, Goustouridis D, Oikonomou P, Chatzandroulis S, Sanopoulou M and Raptis I 2009 Capacitive sensor arrays with controllable deposition of the sensing polymer area for VOCs applications: Design and measurement considerations Procedia Chemistry 1 176-179.

62. Valamontes E, Patsis G P, Goustouridis D, Oikonomou P, Botsialas A, Raptis I, Sanopoulou M 2012 Real time detection of volatile organic compounds through a chemocapacitor system Proc. IMCS 2012 - The 14th International Meeting on Chemical Sensors pp. 1787-1789. 
63. Botsialas A, Oikonomou P, Goustouridis D, Ganetsos Th., Raptis I and Sanopoulou M A 2013 Miniaturized chemocapacitor system for the detection of volatile organic compounds Sens. Actuators B Chem. 177 776-784.

64. Oikonomou P, Raptis I and Sanopoulou M 2014 Monitoring and evaluation of alcoholic fermentation processes using a chemocapacitor sensor array Sensors 14 16258-16273.

65. Emadi T A, Shafai C, Thomson D J, Freund M S, White N D G and Jayas D S 2011 Polymerbased micromachined chemicapacitor gas sensor on a temperature controlled platform Proc. IEEE Sensors, Limerick, 28-31 Oct. 2011, pp. 1024-1027.

66. Emadi T A, Shafai C, Thomson D J, Freund M S, White N D G and Jayas D S 2013 Polymerbased chemicapacitor sensor for 1-octanol and relative humidity detections at different temperatures and frequencies IEEE Sensors Journal 13 519-527.

67. Germain $\mathrm{M}$ E and Knapp M J Optical explosives detection: From color changes to fluorescence turn-on. Chemical Society Reviews 38 2543-2555.

68. Smith R G, D'Souza N and Nicklin S 2008 A review of biosensors and biologically inspired systems for explosives detection. The Analyst 133 571-584.

69. Patel S V, Hobson S T, Cemalovic S and Mlsna T E 2005 Chemicapacitive microsensors for detection of explosives and TICs. Proc. SPIE $598659860 \mathrm{M}-1$.

70. Vobecka Z, Blue R, Vilela F, Skabara P J and Uttamchandani D 2012 Microelectrode sensor utilising nitro-sensitive polymers for application in explosives detection. Micro \& Nano Lett. 7 962964.

71. Stringer J and Derby B 2009 Limits to feature size and resolution in inkjet printing Journal of the European Ceramic Society 29 913-918.

72. Blue R, Vobecka Z, Skabara P J and Uttamchandani D 2013 The development of sensors for volatile nitro-containing compounds as models for explosives detection Sens. Actuators B Chem. 176 534-542.

73. Blue R, Uttamchandani D, Thomson N and Skabara P J 2015 Novel polymer materials for low-cost nitro vapor detection sensors Proc. IEEE Sensors 2015, Busan, South Korea, November 1-4, paper 1128.

74. Chambe P, Canova B, Balabanian A, Pele M and Coeur N 2014 Optimisation of energy harvesting systems for RFID applications Int. J. Computer, Electrical, Automation, Control and Information Eng. 8 1147-1150.

75. Hong S S B, Ibrahim R, Khir M H Md., Zakariya M A and Daud H 2013 Wi-Fi energy harvester for low power RFID application Progress in Electromagnetics Research C 40 69-81.

76. Ong K G and Grimes C A 2001 A carbon nanotube-based sensor for $\mathrm{CO}_{2}$ monitoring Sensors 1 193-205.

77. Ong K G and Grimes C A 2000 A resonant printed-circuit sensor for remote query monitoring of environmental parameters Smart Mater. Struct. 9 421-428.

78. Puligundla P, Jung J and Ko S 2012 Carbon dioxide sensors for intelligent food packaging applications Food Control 25 328-333.

79. Chen W T, Stewart K M E, Carroll J, Mansour R, Abdel-Rahman E and Penlidis A 2013 Novel gaseous phase ethanol sensor implemented with underloaded RF resonator for sensor- 
embedded passive chipless RFIDs Proc. 17th Int. Conf. on Solid-State Sensors, Actuators and Microsystems (Transducers \& Eurosensors XXVII), Barcelona, Spain, 16-20 June, pp. 2059-2062.

80. Oprea A, Courbat J, Bârsan N, Briand D and de Rooij N F 2009 Weimar, U. Temperature, humidity and gas sensors integrated on plastic foil for low-power applications. Sens. Actuators B Chem. 140 227-232.

81. Ishihara T, Kometani K, Hashida M and Takita Y 1991 Application of mixed oxide capacitor to the selective carbon dioxide sensor J. Electrochem. Soc. 138 173-176.

82. Balkus Jr. K J, Ball L J, Gnade B E and Anthony J M 1997 A capacitance type chemical sensor based on $\mathrm{ALPO}_{4}-5$ Molecular Sieves Chem. Mater. 9 380-386.

83. Zampetti E, Maiolo L, Pecora A, Maita F, Pantalei S, Minotti A, Valletta A, Cuscunà M, Macagnano A, Fortunato G and Bearzotti A 2011 Flexible sensorial system based on capacitive chemical sensors integrated with readout circuits fully fabricated on ultra-thin substrate Sens. Actuators B Chem. 155 768-774.

84. Balachandran M D, Shrestha S, Agarwal M, Lvov Y and Varahramyan K $2008 \mathrm{SnO}_{2}$ capacitive sensor integrated with microstrip patch antenna for passive wireless detection of ethylene gas Elect. Lett. 44 464-466.

85. Chatzandroulis S, Tegou E, Goustouridis D, Polymenakos S and Tsoukalas D 2004 Capacitive-type chemical sensors using thin silicon/polymer bimorph membranes Sens. Actuators B Chem. 103 392-396.

86. Patel S V, Mlsna T E, Fruhberger B, Klaassen E, Cemalovic S and Baselt D R 2003 Chemicapacitive microsensors for volatile organic compound detection Sens. Actuators B Chem. 96 541-553.

87. Mlsna T E, Cemalovic S, Warburton M, Hobson S T, Mlsna D A and Patel S V 2006 Chemicapacitive microsensors for chemical warfare agent and toxic industrial chemical detection Sens. Actuators B Chem. 116 192-201.

88. Patel S V, Hobson S T, Cemalovic S and Mlsna T E 2008 Detection of methyl salicylate using polymer-filled chemicapacitors Talanta 76 872-877.

89. McCorkle D L, Warmack R J, Patel S V, Mlsna T, Hunter S R and Ferrell T L 2005 Ethanol vapor detection in aqueous environments using micro-capacitors and dielectric polymers Sens. Actuators B Chem. 107 892-903.

90. Hobson S T, Cemalovic S and Patel S V 2012 Preconcentration and detection of chlorinated organic compounds and benzene Analyst 137 1284-1289.

91. Patel S V and Tolley W K 2014 Developments toward a low-cost approach for long-term, unattended vapor intrusion monitoring. Analyst 139 3770-3780.

92. Vernier Mini Gas Chromatograph. Available online: http://www.vernier.com/products/sensors/gc-mini.

93. SeaPORT Mini GC, Model 510-TC-A. Available online: http://www.seacoastscience.com/Downloads/SeaPORT\%20miniGC.pdf.

94. Locklin J and Bao Z 2006 Effect of morphology on organic thin film transistor sensors Anal. Bioanal. Chem. 384 336-342.

95. Yinon J 2003 Detection of Explosives by Electronic Noses Anal. Chem. 75 98A-105A. 